Article

\title{
Phytochemical Diversity in Rhizomes of Three Reynoutria Species and their Antioxidant Activity Correlations Elucidated by LC-ESI-MS/MS Analysis
}

\author{
Izabela Nawrot-Hadzik ${ }^{1}{ }^{(0)}$, Sylwester Ślusarczyk ${ }^{1}{ }^{\circledR}$, Sebastian Granica ${ }^{2}$, Jakub Hadzik ${ }^{3}$ and \\ Adam Matkowski ${ }^{1,4, *}$ \\ 1 Department of Pharmaceutical Biology and Botany, Wroclaw Medical University, 50-367 Wrocław, Poland; \\ izabela.nawrot-hadzik@umed.wroc.pl (I.N.-H.); sylwester.slusarczyk@umed.wroc.pl (S.Ś.) \\ 2 Department of Pharmacognosy and Molecular Foundations of Phytotherapy, Warsaw Medical University, \\ 02-097 Warszawa, Poland; sgranica@wum.edu.pl \\ 3 Department of Dental Surgery, Wroclaw Medical University, 50-425 Wrocław, Poland; \\ jakub.hadzik@umed.wroc.pl \\ 4 Botanical Garden of Medicinal Plants, Wroclaw Medical University, 50-367 Wrocław, Poland \\ * Correspondence: pharmaceutical.biology@wp.eu; Tel.: +487-1784-0497; Fax: +487-1784-0498
}

Academic Editors: Susana M. Cardoso and Alessia Fazio

Received: 25 February 2019; Accepted: 14 March 2019; Published: 21 March 2019

check for updates

\begin{abstract}
The rhizome of Reynoutria japonica is a well-known traditional herb (Hu zhang) used in East Asia to treat various inflammatory diseases, infections, skin diseases, scald, and hyperlipidemia. It is also one of the richest natural sources of resveratrol. Although, it has been recently included in the European Pharmacopoeia, in Europe it is still an untapped resource. Some of the therapeutic effects are likely to be influenced by its antioxidant properties and this in turn is frequently associated with a high stilbene content. However, compounds other than stilbenes may add to the total antioxidant capacity. Hence, the aim of this research was to examine rhizomes of $R$. japonica and the less studied but morphologically similar species, $R$. sachalinensis and $R$. $\mathrm{x}$ bohemica for their phytochemical composition and antioxidant activity and to clarify the relationship between the antioxidant activity and the components by statistical methods. HPLC/UV/ESI-MS studies of three Reynoutria species revealed 171 compounds, comprising stilbenes, carbohydrates, procyanidins, flavan-3-ols, anthraquinones, phenylpropanoids, lignin oligomers, hydroxycinnamic acids, naphthalenes and their derivatives. Our studies confirmed the presence of procyanidins with high degree of polymerization, up to decamers, in the rhizomes of $R$. japonica and provides new data on the presence of these compounds in other Reynoutria species. A procyanidin trimer digallate was described for the first time in, the studied plants. Moreover, we tentatively identified dianthrone glycosides new for these species and previously unrecorded phenylpropanoid disaccharide esters and hydroxycinnamic acid derivatives. Furthermore, compounds tentatively annotated as lignin oligomers were observed for the first time in the studied species. The rhizomes of all Reynoutria species exhibited strong antioxidant activity. Statistical analysis demonstrated that proanthocyanidins should be considered as important contributors to the total antioxidant capacity.
\end{abstract}

Keywords: proanthocyanidins; Polygoni cuspidati rhizoma; mass spectrometry; stilbenoids

\section{Introduction}

In East Asia, the rhizome of Reynoutria japonica Houtt. (syn. Fallopia japonica [Houtt.] Ronse Decr., obsolete syn. Polygonum cuspidatum Sieb. \& Zucc.) is a well-known traditional herb (Hu zhang, Polygoni cuspidatae rhizoma) used to treat various inflammatory diseases, infections, skin diseases, 
scald, hyperlipidemia, etc. [1]. It is also one of the richest natural source of resveratrol (free and glycosylated) which has proven antioxidant activity [2]. In Europe, $R$. japonica has until recently been considered primarily as a troublesome invasive species that threatens native vegetation. However, rhizome of R. japonica (Polygoni cuspidati rhizoma) has been recently included in the European Pharmacopoeia [3] along with many other traditional Chinese herbs. A morphologically similar species from this genus-R. sachalinensis (F. Schmidt) Nakai, (syn. F. sachalinensis (F. Schmidt) Ronse Decr., P. sachalinense F. Schmidt) and a hybrid between them, $R$. x bohemica Chrtek \& Chrtková (syn. F. $\mathrm{x}$ bohemica (Chrtek \& Chrtková) J.P. Bailey) are not considered as equivalent medicinal plants. Both are also noxious invasive weeds outside their native distribution areas, However, $R$. sachalinensis has been to some extent used traditionally as a herbal medicine in Japan and China for treatment of arthralgia, jaundice, amenorrhea, coughs, scalds and burns, traumatic injuries, carbuncles and sores [4]. Earlier studies revealed striking differences in the metabolic profiles of these three species. $R$. sachalinensis rhizomes contained lower quantities of anthraquinones than rhizomes of $R$. japonica and significantly higher amounts of phenylpropanoid-derived disaccharide esters. Moreover, no stilbenes were detected in $R$. sachalinensis, in contrast to the rich presence of these compounds in rhizomes of $R$. japonica. The phytochemical profile of $R$. x bohemica was intermediate between the two parent species [5]. Some of the therapeutic effects of the studied species are likely to be influenced by their antioxidant properties and this in turn is frequently associated with a high stilbene content [6]. However, some researchers showed no correlation between the content of resveratrol or emodin and antioxidant activity in the obtained extracts and fractions from $R$. japonica [7]. Ding et al. [8] revealed a high positive correlation value for flavanol gallate, resveratrol and catechin but low for piceid, questin (or physcion), and no correlation to emodin-8-O-glucoside, questin (or physcion) and emodin. Moreover, in a study by Pan et al. [9], an ethanol extract of $R$. japonica had a stronger antioxidant activity than resveratrol. These data suggested that compounds other than stilbenes may contribute to the total antioxidant capacity. It is thus worth looking more closely at the phytochemical profile of rhizomes from all three Reynoutria species.

The aim of the present study was to examine rhizomes of the invasive Reynoutria species from the wild population in Poland for their phytochemical composition and antioxidant activity. To clarify the relationship between the antioxidant activity and compounds present in the extracts and fractions, the statistical analysis was performed involved the LC-MS data and results from antioxidant assays.

\section{Results and Discussion}

\subsection{Mass Spectra Analysis, Annotation and Identification of Major Constituents in Extracts and Fractions}

The LC-MS analysis studies of three Reynoutria species ( $R \mathrm{j}, \mathrm{Rs}, \mathrm{Rb})$ revealed a total of 171 detectable compounds, comprising stilbenes, carbohydrates, procyanidins, flavan-3-ols, anthraquinones, phenylpropanoids, lignin oligomers, hydroxycinnamic acids, naphthalenes and their derivatives. Among the detected chromatographic peaks, 37 remained unassigned and without clear indication of their (phyto)chemical nature and four were tentatively defined as carbohydrates. However, most of the unidentified peaks had UV spectra typical for either hydroxycinnamic (the early eluting) or anthraquinone (late eluting) derivatives. Tentative assignments were carried out based on the MS, $\mathrm{MS}^{2}$ and $\mathrm{MS}^{3}$ spectra obtained for major $m / z$ signals recorded in negative ion mode. Further, analysis of UV-vis spectra of compounds and comparison with literature data were used for identification (Figures 1-3 and Table 1). 


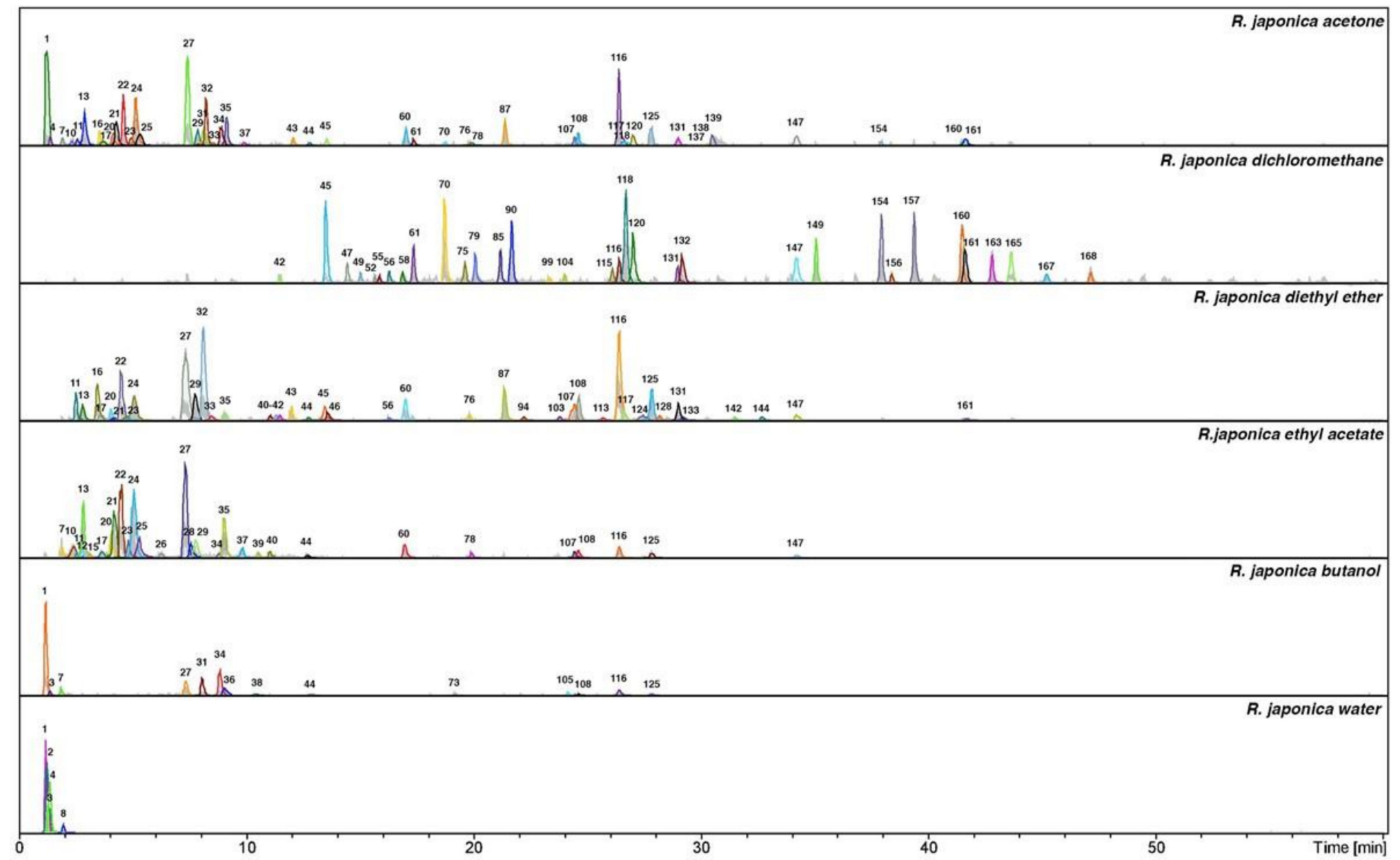

Figure 1. Total ion chromatograms in negative ionization mode and dissect chromatograms of Reynoutria japonica extract and fractions. Deconvolution of an LC/MS mass chromatogram was carried out by using the Bruker's Dissect algorithm. Peak numbers are explained in Table 1. 


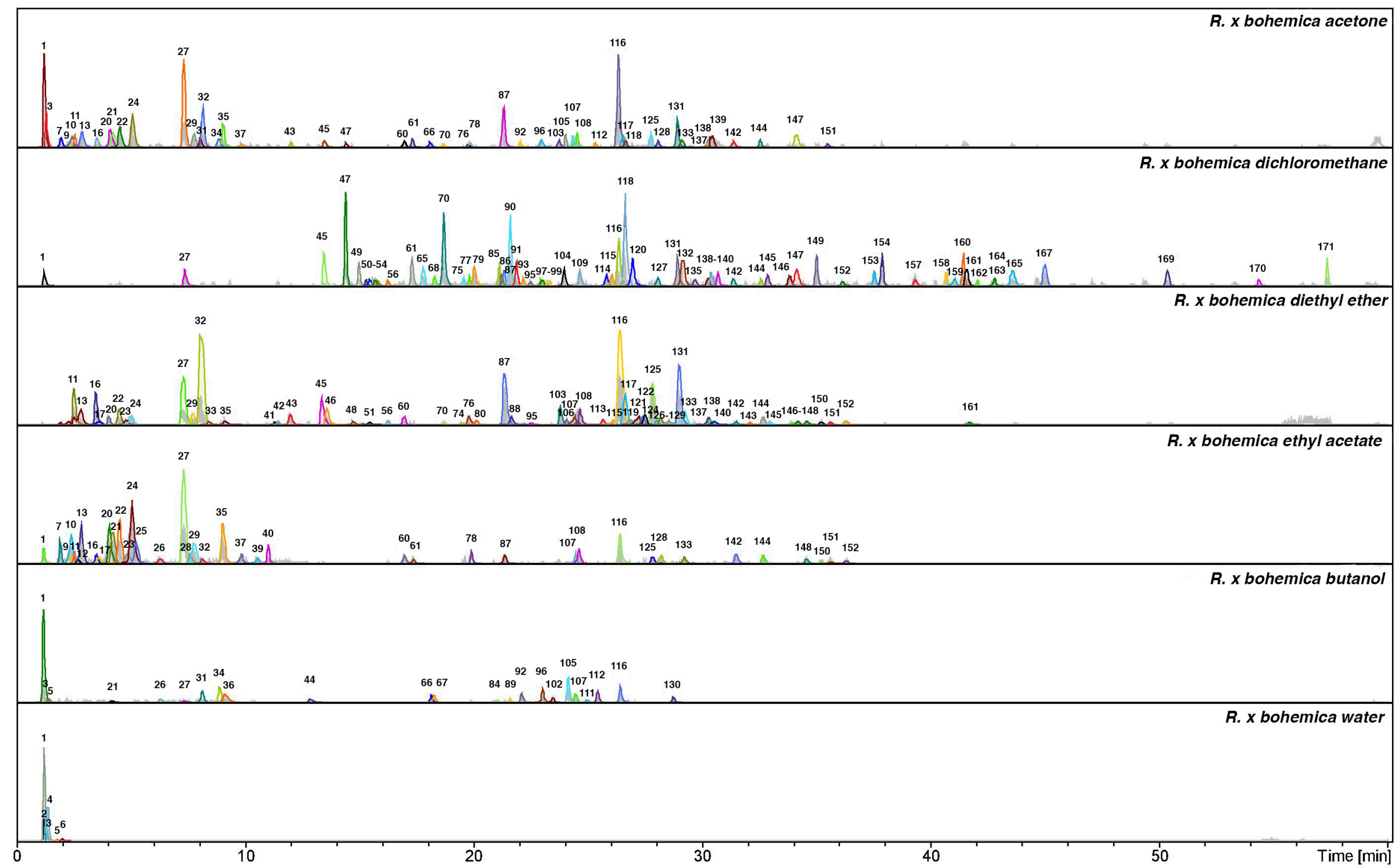

Figure 2. Total ion chromatograms in negative ionization mode and dissect chromatograms of Reynoutria $\mathrm{x}$ bohemica extract and fractions. Deconvolution of an LC/MS mass chromatogram was carried out by using the Bruker's Dissect algorithm. Peak numbers are explained in Table 1. 


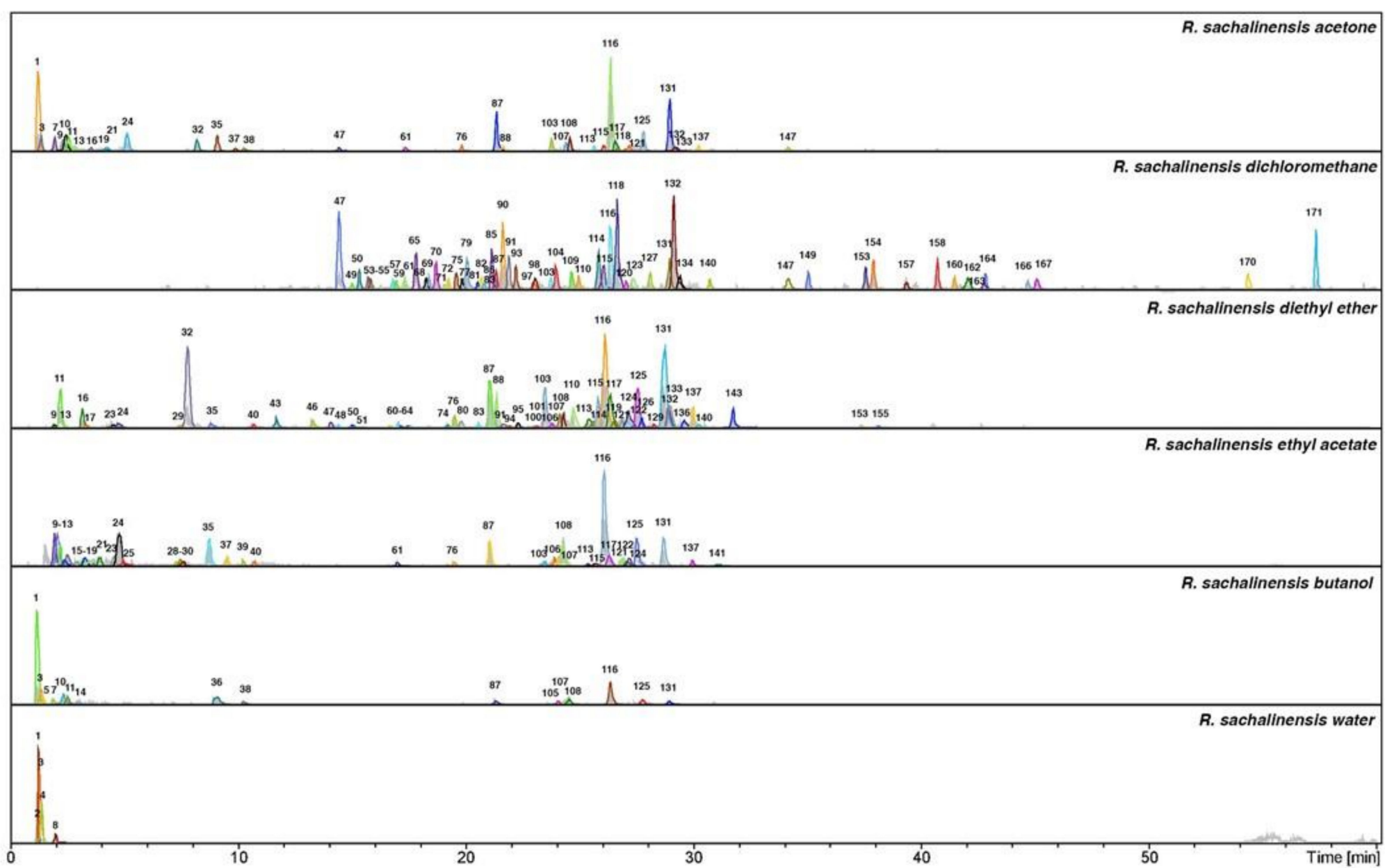

Figure 3. Total ion chromatograms in negative ionization mode and dissect chromatograms of Reynoutria sachalinensis extract and fractions. Deconvolution of an LC/MS mass chromatogram was carried out by using the Bruker's Dissect algorithm. Peak numbers are explained in Table 1. 
Table 1. Retention times, MS data, and UV $\lambda_{\max }$ values of the constituents detected in the extracts and fractions of the three Reynoutria species.

\begin{tabular}{|c|c|c|c|c|c|c|c|c|}
\hline Nr. & Identification & Rt & $\lambda \max (\mathrm{nm})$ & $m / z[\mathrm{M}-\mathrm{H}]^{-}$ & $\mathrm{MS}^{2}$ ions & MS $^{3}$ ions & NL Da & References \\
\hline 1 & $\begin{array}{l}\text { Unknown carbohydrate/e.g., } \\
\text { Disaccharide-sucrose }\end{array}$ & 1.2 & ND & 341.15 & $178.82 b$ & $160.81 b, 142.78$ & 162 & {$[10]$} \\
\hline 2 & Unknown carbohydrate & 1.21 & ND & 683.18 & $341.04 \mathrm{~b}$ & & & \\
\hline 3 & Unknown carbohydrate & 1.3 & ND & 781.12 & $439.02 b$ & $\begin{array}{l}420.95,341.09[\mathrm{M}-2 \mathrm{H}]^{2-} \\
277.01 \mathrm{~b}, 178.80\end{array}$ & 162 & \\
\hline 4 & Unknown carbohydrate & 1.4 & ND & 781.12 & $439.04 b$ & $\begin{array}{l}421.04,340.98[\mathrm{M}-2 \mathrm{H}]^{2-} \mathrm{b} \\
276.87,178.83\end{array}$ & 162 & \\
\hline 5 & Galloyl-glucose & 1.5 & 210,276 & 331.13 & $270.72,168.58 \mathrm{~b}$ & & & [11] \\
\hline 6 & Unknown & 1.8 & $235,275,325$ & 477.1 & $459.05 b, 357.04,234.83,150.80$ & & & \\
\hline 7 & Procyanidin dimer, Type B & 1.9 & 225,280 & 577.11 & $\begin{array}{l}559.04,450.99,424.96 b, 407.15 \\
288.93,286.97\end{array}$ & $406.90 \mathrm{~b}, 381.02,272.85$ & 152 & {$[11-13]$} \\
\hline 8 & Unknown & 2.0 & $235,275,325$ & $439.00 \mathrm{~b}, \mathbf{4 2 5 . 0 5}$ & $344.98,240.80 \mathrm{~b}$ & & & \\
\hline 9 & Procyanidin dimer, Type B & 2.3 & 225,280 & 577.13 & $\begin{array}{l}559.04,450.97,424.95 b, 407.09 \\
288.93,286.97\end{array}$ & $406.91 \mathrm{~b}, 381.12,339.07,272.90$ & 152 & {$[11-13]$} \\
\hline 10 & Procyanidin trimer, Type B & 2.4 & 225,280 & 865.19 & $\begin{array}{l}739.14,695.12 b, 577.07 \\
406.98,286.87\end{array}$ & & & {$[12,13]$} \\
\hline 11 & Catechin* & 2.6 & 225,280 & 288.99 & $270.90,244.91 b, 204.85,178.83$ & & & \\
\hline 12 & $\begin{array}{l}\text { Procyanidin trimer } \\
\text { monogallate }\end{array}$ & 2.7 & 225,280 & 1017.2 & $\begin{array}{l}891.18,865.18,847.12,729.12 b \\
577.11,407.07,287.81\end{array}$ & & & [13] \\
\hline 13 & Procyanidin dimer, Type B & 2.8 & 225,280 & 577.08 & $\begin{array}{l}559.05,451.00,424.96 b, 407.00 \\
288.90,286.97\end{array}$ & $406.90 \mathrm{~b}, 381.11,272.87$ & 152 & [11-13] \\
\hline 14 & Procyanidin pentamer & 3.1 & 225,280 & $720.55[\mathrm{M}-2 \mathrm{H}]^{2-}$ & $\begin{array}{l}1315.33,1151.29 \mathrm{~b}, 1027.23,863.22 \\
635.05,577.05,288.85\end{array}$ & & & [12] \\
\hline 15 & Procyanidin trimer, Type B & 3.2 & 225,280 & 865.21 & $\begin{array}{l}739.13,695.14 b, 577.08,407.00 \\
286.90\end{array}$ & & & {$[12,13]$} \\
\hline 16 & Epicatechin * & 3.5 & 225,280 & 288.82 & $\begin{array}{l}270.76,244.75 b, 230.68 \\
204.70,178.65\end{array}$ & & & \\
\hline 17 & $\begin{array}{l}\text { Procyanidin dimer } \\
\text { monogallate }\end{array}$ & 3.6 & 225,280 & 729.17 & $577.06 b, 425.06,407.07,286.92$ & $\begin{array}{l}559.05,450.98,424.98 \\
407.00 \mathrm{~b}, 288.90\end{array}$ & 152 & {$[11,13]$} \\
\hline 18 & $\begin{array}{l}\text { Procyanidin trimer } \\
\text { monogallate }\end{array}$ & 3.7 & 225,280 & 1017.2 & $\begin{array}{l}\text { 865.16b, 847.15, 729.11, } \\
577.06,406.97\end{array}$ & $\begin{array}{l}847.14,695.12 b, 577.05 \\
394.95,286.81\end{array}$ & 152 & [13] \\
\hline 19 & Procyanidin trimer, Type B & 4.0 & 225,280 & 865.2 & $\begin{array}{l}739.15,695.14 \mathrm{~b}, 577.07 \\
406.99,286.89\end{array}$ & & & {$[12,13]$} \\
\hline 20 & Piceatannol glucoside * & 4.1 & $220,305,318$ & 405.06 & $242.73 b$ & $\begin{array}{l}224.70 b, 214.68,200.69, \\
184.64,174.73\end{array}$ & 162 & \\
\hline 21 & Procyanidin trimer, Type B & 4.2 & 225,280 & 865.19 & $\begin{array}{l}739.15,695.12 b, 577.08 \\
406.98,286.87\end{array}$ & & & {$[12,13]$} \\
\hline
\end{tabular}


Table 1. Cont.

\begin{tabular}{|c|c|c|c|c|c|c|c|c|}
\hline Nr. & Identification & Rt & $\lambda \max (\mathrm{nm})$ & $m / z[\mathrm{M}-\mathrm{H}]^{-}$ & MS $^{2}$ ions & $\mathrm{MS}^{3}$ ions & NL Da & References \\
\hline 22 & Resveratroloside* & 4.6 & $219,304,315$ & $389.07,435.13\left[\mathrm{M}+\mathrm{HCOO}^{-}\right.$ & $389.07 \mathrm{~b}, 226.91$ & & & \\
\hline 23 & $\begin{array}{l}\text { Procyanidin trimer } \\
\text { monogallate }\end{array}$ & 4.8 & 225,280 & 1017.19 & $\begin{array}{l}\text { 865.14, 847.15, 729.16b, 603.09, } \\
559.08,407.06,288.89\end{array}$ & $\begin{array}{l}847.07 \mathrm{~b}, 695.02,575.94 \\
451.02,286.80\end{array}$ & 152 & [13] \\
\hline 24 & $\begin{array}{l}\text { Procyanidin dimer } \\
\text { monogallate }\end{array}$ & 5.1 & 225,280 & 729.12 & $\begin{array}{l}577.05,559.05,451.00,441.01, \\
407.02 \mathrm{~b}, 288.90\end{array}$ & $559.01 \mathrm{~b}, 450.99,406.95,288.86$ & 152 & {$[11,13]$} \\
\hline 25 & Procyanidin tetramer, Type B & 5.3 & 225,280 & 1153.26 & $\begin{array}{l}\text { 1001.20, 983.20, 865.16b, 739.12, } \\
575.09,449.02\end{array}$ & $983.18 b, 804.93,533.18,382.95$ & 152 & {$[12,13]$} \\
\hline 26 & Procyanidin pentamer & 6.3 & 225,280 & $720.55[\mathrm{M}-2 \mathrm{H}]^{2-}$ & $\begin{array}{l}1315.33,1151.29 b, 1027.23,863.22, \\
635.05,577.05 .288 .85\end{array}$ & & & [12] \\
\hline 27 & Piceid * & 7.4 & $218,308,318$ & 389.12, 435.07 [M + HCOO $]^{-}$ & $226.71 \mathrm{~b}$ & & & \\
\hline 28 & Procyanidin trimer digallate & 7.6 & 225,280 & 1169.24 & $\begin{array}{l}1151.24,999.21,881.22 b, 729.18 \\
603.11,406.98\end{array}$ & & & [13] \\
\hline 29 & $\begin{array}{l}\text { Procyanidin dimer digallate, } \\
\text { Type B }\end{array}$ & 7.7 & 225,280 & 881.16 & 729.12b, 559.08, 407.01, 288.86 & $\begin{array}{l}\text { 603.07, 577.08, 559.07, } \\
451.01 b, 407.10,288.98\end{array}$ & 152 & [13] \\
\hline 30 & $\begin{array}{l}\text { Procyanidin trimer } \\
\text { monogallate }\end{array}$ & 7.9 & 225,280 & 1017.22 & $\begin{array}{l}\text { 865.17, 847.15, 729.13b, 603.09, } \\
575.09,406.98,286.88\end{array}$ & $\begin{array}{l}847.07 \mathrm{~b}, 739.12,714.02, \\
577.04,448.84,288.69\end{array}$ & 152 & [13] \\
\hline 31 & Procyanidin heptamer & 8.1 & 225,280 & $\begin{array}{l}1008.63\left[\mathrm{M}-2 \mathrm{H}^{-}, 1152.74,\right. \\
1021.26,999.18,631.20 \\
567.10,499.09 \mathrm{~b}\end{array}$ & $\begin{array}{l}\text { 484.00b, } 452.98,419.04,345.92 \\
314.85,288.79\end{array}$ & & & {$[12,14]$} \\
\hline 32 & Epicatechin-3-O-gallate * & 8.2 & 220,280 & 440.95 & $\begin{array}{l}330.82,302.80,288.82 b, 270.81 \\
244.82\end{array}$ & & & \\
\hline 33 & Procyanidin dimer, Type B & 8.4 & 225,280 & 577.09 & $\begin{array}{l}559.08,450.96, \mathbf{4 2 4 . 9 4 b}, 407.06 \\
288.92\end{array}$ & $406.91 b, 381.04,339.00,272.85$ & 152 & [11-13] \\
\hline 34 & Procyanidin octamer & 8.8 & 225,280 & $\begin{array}{l}1152.70[\mathrm{M}-2 \mathrm{H}]^{-}, 901.21 \\
879.11,507.02,439.04 \mathbf{b}\end{array}$ & $423.93 \mathrm{~b}, 392.86,358.98,315.84$ & & & [15] \\
\hline 35 & $\begin{array}{l}\text { Procyanidin trimer } \\
\text { monogallate }\end{array}$ & 9.0 & 225,280 & 1017.21 & $\begin{array}{l}\text { 865.12, } 847.15,729.15 b, 603.08 \\
406.99,288.90\end{array}$ & $\begin{array}{l}847.15 b, 684.05,518.85 \\
451.83,395.07,301.69\end{array}$ & 152 & [13] \\
\hline 36 & Procyanidin octamer & 9.1 & 225,280 & $\begin{array}{l}1152.69[\mathrm{M}-2 \mathrm{H}]^{2-}, 901.16 \\
864.10,845.10,439.02 \\
\mathbf{3 8 2 . 9 0 b}\end{array}$ & $302.80 \mathrm{~b}, 284.83,176.67$ & & & [15] \\
\hline 37 & $\begin{array}{l}\text { Procyanidin tetramer } \\
\text { monogallate }\end{array}$ & 9.8 & 225,280 & $652.11[M-2 H]^{2-} b, 1305.32$ & $\begin{array}{l}\text { 1179.22, 1017.23, 863.18, 729.11, } \\
\text { 576.00, 567.07b, 440.99, 288.88, } \\
286.86\end{array}$ & & & [15] \\
\hline 38 & Unknown & 10.3 & 225,280 & $1532.47, \mathbf{3 8 2 . 9 0 b}$ & $302.77 \mathrm{~b}, 284.88,178.67$ & & & \\
\hline 39 & Procyanidin gallate & 10.5 & 225,280 & $\begin{array}{l}796.27[\mathrm{M}-3 \mathrm{H}]^{3} \mathrm{~b}, 948.11 \\
{[\mathrm{M}-2 \mathrm{H}]^{2}}\end{array}$ & $\begin{array}{l}1467.40,1305.34,1179.31,1017.21 \\
863.17,729.15 b, 440.96,288.86\end{array}$ & & & [15] \\
\hline 40 & $\begin{array}{l}\text { Procyanidin trimer } \\
\text { monogallate }\end{array}$ & 11.0 & 225,280 & 1017.2 & $\begin{array}{l}\text { 891.16, 847.16, 729.14b, 603.07, } \\
559.05,407.03,288.87\end{array}$ & & & [13] \\
\hline 41 & Procyanidin gallate & 11.3 & 225,280 & $660.32,505.17 \mathrm{~b}$ & $\begin{array}{l}1151.19,999.17,881.14,584.04 b, \\
440.96,302.86\end{array}$ & & & {$[12,13,15]$} \\
\hline
\end{tabular}


Table 1. Cont.

\begin{tabular}{|c|c|c|c|c|c|c|c|c|}
\hline Nr. & Identification & $\mathbf{R t}$ & $\lambda \max (\mathrm{nm})$ & $m / z[\mathbf{M}-\mathbf{H}]^{-}$ & MS $^{2}$ ions & MS $^{3}$ ions & NL Da & References \\
\hline 42 & Resveratrol-hexoside & 11.4 & $219,304,315$ & 389.06 & 226.71 & & & \\
\hline 43 & $\begin{array}{l}\text { Procyanidin dimer } \\
\text { monogallate }\end{array}$ & 12.0 & 225,280 & 729.13 & $\begin{array}{l}711.11,603.05,577.04,559.05 \\
407.01 b, 288.91\end{array}$ & $559.03,450.97,406.93 b, 288.85$ & 152 & {$[11,13]$} \\
\hline 44 & Emodin glucoside * & 12.8 & $\begin{array}{l}220,247,269, \\
281,423\end{array}$ & 431.3 & $268.75 b$ & $239.63,226.68,224.72 b$ & 162 & \\
\hline 45 & Resveratrol * & 13.5 & $218,306,318$ & 226.78 & $184.60,158.67 \mathrm{~b}, 142.68$ & & & \\
\hline 46 & $\begin{array}{l}\text { Procyanidin dimer digallate, } \\
\text { Type B }\end{array}$ & 13.6 & 225,280 & 881.13 & 729.11b, 559.12, 407.05, 288.90 & $\begin{array}{l}\text { 603.07, 577.08, 559.04, } \\
451.01 \mathrm{~b}, 407.04,288.98\end{array}$ & 152 & [13] \\
\hline 47 & $N$-trans-feruloyltyramine * & 14.4 & $220,281,323$ & 312.08 & $296.97 \mathrm{~b}, 177.83,134.87$ & & & \\
\hline 48 & Acetyl lapathoside d & 14.7 & $220,290,315$ & 675.24 & $\begin{array}{l}\text { 633.17, 615.12, 529.07b, 511.12, } \\
487.12,453.11,306.97\end{array}$ & $487.04 \mathrm{~b}, 469.19,306.96$ & 146 & [16] \\
\hline 49 & $\mathrm{~N}$-Feruloyl-methoxytyramine & 15.0 & $220,281,323$ & 342.14 & $\begin{array}{l}327.04 b, 308.97,297.01,177.84 \\
134.87\end{array}$ & & & [17] \\
\hline 50 & $\begin{array}{l}\text { Phenylpropanoid-derived } \\
\text { disaccharide ester }\end{array}$ & 15.3 & $220,284,315$ & 655.21 & $\begin{array}{l}\text { 613.18b, 595.18, 571.16, 553.10, } \\
425.12,306.99\end{array}$ & & & \\
\hline 51 & Cyanidin & 15.4 & $210,286,332$ & 286.9 & $\begin{array}{l}268.79,150.59 b, 134.71,124.75 \\
106.72\end{array}$ & & & [18] \\
\hline 52 & Unknown & 15.6 & $225,287,315$ & 585.28 & $537.17 \mathrm{~b}, 371.13,359.13$ & & & \\
\hline 53 & Unknown & 15.7 & $225,287,315$ & 583.27 & $535.23 \mathrm{~b}, 369.10,357.25,194.91$ & & & \\
\hline 54 & Unknown & 15.8 & 220,280 & 685.23 & $\begin{array}{l}\text { 643.20b, 625.20, 601.19, 337.12, } \\
192.90\end{array}$ & & & \\
\hline 55 & Unknown & 15.9 & $220,287,315$ & 585.29 & $537.20 \mathrm{~b}, 359.12,345.14$ & & & \\
\hline 56 & Torachrysone glucoside * & 16.2 & $225,267,325$ & 407.16 & $244.87 \mathrm{~b}$ & 229.97 & 162 & \\
\hline 57 & Unknown & 16.8 & 225,280 & 371.12 & $327.08 b, 297.08$ & & & \\
\hline 58 & Unknown & 16.85 & 225,280 & 597.26 & $\begin{array}{l}553.13,549.23,383.11 b, 371.12 \\
194.86\end{array}$ & & & \\
\hline 59 & Unknown & 16.9 & 225,280 & 583.31 & $553.20,369.11 b, 357.15,194.80$ & & & \\
\hline 60 & Emodin glucoside * & 17.0 & $\begin{array}{l}221,247,269, \\
281,423\end{array}$ & 431.04 & $310.84,292.76,268.75 b$ & $264.71,240.73,224.70 \mathrm{~b}$ & 162 & \\
\hline 61 & $\begin{array}{l}\text { Dihydroksyferuloyl-O- } \\
\text { acetoxy-p-coumaroyl-O- } \\
\text { caffeoylquinic acid }\end{array}$ & 17.4 & $214,282,325$ & 735.27 & $693.19, \mathbf{5 5 9 . 1 2 b}, 541.18$ & $\begin{array}{l}517.11 b, 499.10,337.04, \\
264.90,192.83\end{array}$ & 176 & [18] \\
\hline 62 & Tatariside e & 17.5 & $220,290,315$ & 717.39 & $\begin{array}{l}675.19,571.11 b, 529.20,453.12 \\
288.94\end{array}$ & $529.06 \mathrm{~b}, 511.05,469.03,306.85$ & 146 & [19] \\
\hline 63 & Tatariside e & 17.7 & $220,290,315$ & 717.4 & $\begin{array}{l}675.19,571.13 b, 529.24,453.10 \\
288.93\end{array}$ & $529.05 b, 511.05,469.00,306.85$ & 146 & [19] \\
\hline 64 & Unknown & 17.8 & 225,280 & 314.95 & $\begin{array}{l}299.78 b, 270.98,246.72,204.68 \\
178.78\end{array}$ & & & \\
\hline 65 & $\begin{array}{l}\text { (diacetoxy-methoxyphenyl) } \\
\text { acroyl-O-p-coumaroyl-O- } \\
\text { caffeoylquinic acid }\end{array}$ & 17.9 & $214,282,325$ & 777.25 & $\begin{array}{l}\text { 735.22b, } 717.25,693.00, \mathbf{6 0 1 . 1 6} \\
559.13,337.09\end{array}$ & $559.13 b, 541.11,499.05$ & 176 & {$[18]$} \\
\hline
\end{tabular}


Table 1. Cont.

\begin{tabular}{|c|c|c|c|c|c|c|c|c|}
\hline Nr. & Identification & $\mathbf{R t}$ & $\lambda \max (\mathrm{nm})$ & $m / z[\mathbf{M}-\mathbf{H}]^{-}$ & MS $^{2}$ ions & MS $^{3}$ ions & NL Da & References \\
\hline 66 & $\begin{array}{l}\text { Emodin } \\
\text { bianthrone-hexose-(malonic } \\
\text { acid)-hexose }\end{array}$ & 18.1 & 220,278 & 919.21 & $\begin{array}{l}875.23,757.10,713.20 \mathrm{~b}, 671.25 \\
509.08,458.00\end{array}$ & $\begin{array}{l}713.15 b, 509.04,502.00 \\
457.99,253.79\end{array}$ & 162 & {$[20,21]$} \\
\hline 67 & $\begin{array}{l}\text { Derivative of Emodin } \\
\text { bianthrone-hexose-malonic } \\
\text { acid }\end{array}$ & 18.2 & 220,278 & 1005.23 & $\begin{array}{l}961.13,917.29,757.10,713.23 \mathrm{~b} \\
458.10\end{array}$ & & & [21] \\
\hline 68 & Unknown & 18.3 & $225,280,325$ & 811.36 & $\begin{array}{l}\text { 793.32b, 763.38, 745.34, 669.23, } \\
567.21,389.09,342.99,311.93\end{array}$ & & & \\
\hline 69 & Unknown & 18.4 & $225,280,325$ & 597.27 & $\begin{array}{l}549.18 b, 401.11,357.12,342.12, \\
194.87\end{array}$ & & & \\
\hline 70 & $\begin{array}{l}\text { (diacetoxy-methoxyphenyl) } \\
\text { acroyl-O-p-coumaroyl-O- } \\
\text { caffeoylquinic acid }\end{array}$ & 18.7 & $214,282,325$ & 777.26 & $\begin{array}{l}\text { 735.24b, 717.25, 693.00, 601.16, } \\
559.20,337.04\end{array}$ & $559.13 b, 541.17,499.13$ & 176 & [18] \\
\hline 71 & $\begin{array}{l}\text { Trimer lignin } \beta-O-4 \text {-linked } S \\
\text { unit with syringaresinol } \\
{\left[S-\left(\beta-O-4^{\prime}\right)-S-\left(\beta-\beta^{\prime}\right)-S\right]}\end{array}$ & 19.0 & 220,280 & 643.29 & $\begin{array}{l}\text { 613.22, 417.13b, 387.15, 224.93, } \\
194.87\end{array}$ & & & {$[22]$} \\
\hline 72 & $\begin{array}{l}\text { Tetramer lignin, } \\
\text { S-(8-O-4' })-S-\left(8-O-4^{\prime}\right)-S-\left(8-8^{\prime}\right)-S\end{array}$ & 19.1 & 220,280 & 869.39 & $\begin{array}{l}\text { 851.34b, 821.34, 697.27, 643.22, } \\
595.21,417.15,387.15\end{array}$ & & & [22] \\
\hline 73 & Emodin-O-(sulfonyl)-glucoside & 19.2 & 214,280 & 511 & $430.99,268.73 b, 240.74,224.96$ & & & {$[11,20]$} \\
\hline 74 & $\begin{array}{l}\text { Lapathoside c } \\
\text { (diacetoxy-methoxyphenyl) }\end{array}$ & 19.5 & $220,290,315$ & 809.28 & 663.13b, 485.07, 322.98 & $517.04,485.10 \mathrm{~b}, 322.88,280.89$ & 146 & {$[16,23]$} \\
\hline 75 & $\begin{array}{l}\text { (daceytoxy-methoxyphenyl) } \\
\text { caffeoylquinic acid }\end{array}$ & 19.6 & $214,282,325$ & 777.3 & $\begin{array}{l}\text { 735.24b, 717.13, 693.13, 601.17, } \\
559.00,337.10\end{array}$ & $559.13 \mathrm{~b}, 541.13,499.00$ & 176 & [18] \\
\hline 76 & Lapathoside c isomer & 19.7 & $220,290,315$ & 809.28 & 663.13b, $485.07,322.98$ & $517.04,485.10 \mathrm{~b}, 322.88,280.89$ & 146 & {$[16,23]$} \\
\hline 77 & Unknown & 19.8 & $220,280,315$ & 327.26 & $\begin{array}{l}309.12,291.10,228.95 b, 210.95 \\
170.91\end{array}$ & & & \\
\hline 78 & $\begin{array}{l}\text { Emodin-8-O-(6'-O- } \\
\text { malonyl)-glucoside * }\end{array}$ & 19.81 & $220,282,423$ & 517.05 & $472.99 \mathrm{~b}, 431.10$ & & & \\
\hline 79 & Oligolignol-hedyotisol & 20.1 & 220,280 & 809.36 & $\begin{array}{l}\text { 791.33, 773.34, 761.25, 743.33b } \\
565.21,417.11\end{array}$ & & & [24] \\
\hline 80 & Tatariside e & 20.2 & $220,290,315$ & 717.22 & $\begin{array}{l}\text { 675.17, 571.09b, 529.10, 511.17, } \\
487.09\end{array}$ & $529.05 b, 511.04,487.03$ & 146 & [19] \\
\hline 81 & Derivative of lignin- $S(8-8) S$ & 20.5 & 220,280 & 641.32 & $\begin{array}{l}623.22,611.20 \mathrm{~b}, 417.13,387.08 \\
347.09,222.87\end{array}$ & & & [25] \\
\hline 82 & Unknown & 20.7 & $220,280,315$ & 1035.48 & $\begin{array}{l}\text { 1017.45b, 999.38, 969.41, 821.41, } \\
791.35,595.14\end{array}$ & & & \\
\hline 83 & Tatariside a & 20.8 & $220,290,315$ & 759.22 & $\begin{array}{l}\text { 717.21b, 613.13, 571.13, 453.04, } \\
288.94\end{array}$ & $\begin{array}{l}571.09 \mathrm{~b}, 553.10,529.07, \\
511.06,306.71\end{array}$ & 146 & [19] \\
\hline 84 & $\begin{array}{l}\text { Methyl derivative of Emodin } \\
\text { bianthrone-hexose-(malonic } \\
\text { acid)-hexose }\end{array}$ & 21.0 & 220,278 & 933.21 & $889.37 b, 727.21,458.06$ & & & [21] \\
\hline
\end{tabular}


Table 1. Cont

\begin{tabular}{|c|c|c|c|c|c|c|c|c|}
\hline Nr. & Identification & $\mathbf{R t}$ & $\lambda \max (\mathrm{nm})$ & $m / z[\mathbf{M}-\mathbf{H}]^{-}$ & MS $^{2}$ ions & $\mathrm{MS}^{3}$ ions & NL Da & References \\
\hline 85 & $\begin{array}{l}\text { Oligolignol-e.g., } \\
\text { hedyotisol(isomer) }\end{array}$ & 21.1 & 220,280 & 809.37 & $\begin{array}{l}\text { 791.34b, 773.25, 761.31, 743.34, } \\
\text { 565.21, 417.15 }\end{array}$ & & & [24] \\
\hline 86 & $\begin{array}{l}\text { Acetyl derivative of } \\
\text { (diacetoxy-methoxyphenyl) } \\
\text { acroyl-O-p-coumaroyl-O- } \\
\text { caffeoylquinic acid }\end{array}$ & 21.2 & $214,282,325$ & 819.29 & $\begin{array}{l}777.29 b, 759.25,643.19,601.14 \\
513.13\end{array}$ & $601.10 \mathrm{~b}, 583.16,559.07,337.02$ & 176 & [18] \\
\hline 87 & Hydropiperoside * & 21.3 & $220,290,315$ & 779.26 & $\begin{array}{l}\text { 633.16b, 615.19, 487.13, 469.16, } \\
453.09\end{array}$ & $\begin{array}{l}\text { 487.12b, 469.16, 453.11, } \\
307.10,289.03\end{array}$ & 146 & \\
\hline 88 & $\begin{array}{l}\text { (3,6-O-di- }- \text {-coumaroyl)- } \beta- \\
\text { fructofuranosyl- }(2 \rightarrow 1)-\left(2^{\prime}-O-\right. \\
\text { acetyl-6'-O-feruloyl)- } \beta- \\
\text { glucopyranoside }{ }^{*}\end{array}$ & 21.5 & $220,290,315$ & 851.25 & $809.23,705.20 b, 675.20,527.07$ & $\begin{array}{l}663.22 \mathrm{~b}, 645.38,559.16 \\
527.16,485.12\end{array}$ & 146 & \\
\hline 89 & $\begin{array}{l}\text { Derivative of Emodin } \\
\text { bianthrone-di-hexose }\end{array}$ & 21.6 & 220,278 & 1019.22 & $\begin{array}{l}975.25,931.42 b, 889.25,727.18 \\
458.06\end{array}$ & & & [21] \\
\hline 90 & $\begin{array}{l}\text { Acetyl derivative of } \\
\text { (diacetoxy-methoxyphenyl) } \\
\text { acroyl-O-p-coumaroyl-O- } \\
\text { caffeoylquinic acid }\end{array}$ & 21.7 & $214,282,325$ & 819.28 & $\begin{array}{l}777.25 b, 759.38,643.18,601.14 \\
513.13\end{array}$ & $\begin{array}{l}\text { 601.18b, 583.18, 559.15, } \\
541.11,337.02\end{array}$ & 176 & [18] \\
\hline 91 & Unknown & 21.9 & $220,280,315$ & 329.27 & $\begin{array}{l}311.18,293.12,228.95 b, 210.96 \\
170.91\end{array}$ & & & \\
\hline 92 & $\begin{array}{l}\text { Emodin } \\
\text { bianthrone-hexose-(malonic } \\
\text { acid)-hexose }\end{array}$ & 22.0 & 220,278 & 919.2 & $\begin{array}{l}875.24,757.09,713.20 \mathrm{~b}, 671.13 \\
509.06,458.00\end{array}$ & $713.18 b, 508.96,501.88,458.03$ & 162 & {$[20,21]$} \\
\hline 93 & $\begin{array}{l}\text { Oligolignol-e.g.,hedyotisol } \\
\text { (isomer) }\end{array}$ & 22.1 & 220,280 & 809.32 & $\begin{array}{l}\text { 791.30, } 773.25,761.28,743.29 \\
611.20 \mathrm{~b}, 565.18,417.19\end{array}$ & & & {$[24]$} \\
\hline 94 & $\begin{array}{l}\text { Phenylpropanoid-derived } \\
\text { disaccharide ester }\end{array}$ & 22.2 & $220,290,315$ & 987.31 & $\begin{array}{l}969.39 b, 957.50,851.27,823.32, \\
633.18,453.09\end{array}$ & & & \\
\hline 95 & Tatariside a (isomer) & 22.5 & $220,290,315$ & 759.4 & $\begin{array}{l}717.22,675.16,613.14 b, 571.21 \\
529.18\end{array}$ & $571.09 \mathrm{~b}, 553.05,529.06,511.06$ & 146 & [21] \\
\hline 96 & $\begin{array}{l}\text { Emodin } \\
\text { bianthrone-hexose-(malonic } \\
\text { acid)-hexose }\end{array}$ & 23.0 & 220,278 & 919.21 & $\begin{array}{l}\text { 875.23, 757.10, } 713.22 \mathrm{~b}, 671.25 \\
509.09,458.13\end{array}$ & $713.16 \mathrm{~b}, 509.00,501.75,458.20$ & 162 & {$[20,21]$} \\
\hline 97 & Unknown & 23.01 & $220,280,315$ & 837.37 & $\begin{array}{l}819.31,695.25,640.23 b, 579.18 \\
347.02\end{array}$ & & & \\
\hline 98 & $\begin{array}{l}\text { Acetyl derivative of } \\
\text { (diacetoxy-methoxyphenyl) } \\
\text { acroyl-O-p-coumaroyl-O- } \\
\text { caffeoylquinic acid }\end{array}$ & 23.1 & $220,288,325$ & 819.26 & $\begin{array}{l}\text { 777.28b, 759.38, 643.17, 601.25, } \\
513.13,361.01\end{array}$ & 601.13b, 583.13, 559.11, 336.97 & 176 & [18] \\
\hline 99 & $\begin{array}{l}\text { Acetyl derivative of } \\
\text { (diacetoxy-methoxyphenyl) } \\
\text { acroyl-O-p-coumaroyl-O- } \\
\text { caffeoylquinic acid }\end{array}$ & 23.3 & $220,288,325$ & 819.28 & $\begin{array}{l}777.27 \mathrm{~b}, 759.25,643.17,601.25 \\
513.13,361.04\end{array}$ & $601.15 b, 583.10,559.11,336.97$ & 176 & {$[18]$} \\
\hline
\end{tabular}


Table 1. Cont.

\begin{tabular}{|c|c|c|c|c|c|c|c|c|}
\hline Nr. & Identification & $\mathbf{R t}$ & $\lambda \max (\mathrm{nm})$ & $m / z[\mathrm{M}-\mathrm{H}]^{-}$ & MS $^{2}$ ions & MS $^{3}$ ions & NL Da & References \\
\hline 100 & $\begin{array}{l}\text { Isomer of } \\
(3,6-O-\text { di- } p \text {-coumaroyl)-- } \beta- \\
\text { fructofuranosyl- }(2 \rightarrow 1)-\left(2^{\prime}-O-\right. \\
\text { acetyl-6'-O-feruloyl)- } \beta \text { - } \\
\text { glucopyranoside or } \\
\text { tatariside d }\end{array}$ & 23.4 & $220,290,315$ & 851.39 & $809.24,705.19 b, 663.27,527.12$ & $\begin{array}{l}\text { 663.20b, } 645.25,559.13 \\
527.11,485.10\end{array}$ & 146 & [19] \\
\hline 101 & Hydropiperoside isomer & 23.45 & $220,290,315$ & 779.36 & $\begin{array}{l}\text { 633.11b, 615.25, 487.06, 469.13, } \\
453.38,288.86\end{array}$ & $\begin{array}{l}487.06 \mathrm{~b}, 469.18,453.08, \\
306.90,288.88\end{array}$ & 146 & \\
\hline 102 & $\begin{array}{l}\text { Methyl derivative of Emodin } \\
\text { bianthrone-hexose-(malonic } \\
\text { acid)-hexose }\end{array}$ & 23.5 & 220,278 & 933.21 & $889.47 \mathrm{~b}, 727.24,458.09$ & & & [21] \\
\hline 103 & Vanicoside $C^{*}$ & 23.8 & $220,290,315$ & 821.23 & $\begin{array}{l}\text { 761.18, 675.16b, 633.19, 529.10, } \\
487.09,288.87\end{array}$ & $633.15,529.10 \mathrm{~b}, 453.18,288.98$ & 146 & \\
\hline 104 & $\begin{array}{l}\text { Acetyl derivative of } \\
\text { (diacetoxy-methoxyphenyl) } \\
\text { acroyl-O-p-coumaroyl-O- } \\
\text { caffeoylquinic acid }\end{array}$ & 24.0 & $220,290,315$ & 819.31 & $\begin{array}{l}777.29 b, 759.25,643.17,601.25 \\
583.20,361.04\end{array}$ & $601.15 b, 583.10,559.11,337.13$ & 176 & [18] \\
\hline 105 & $\begin{array}{l}\text { Derivative of Emodin } \\
\text { bianthrone-hexose- } \\
\text { malonic acid }\end{array}$ & 24.1 & 220,278 & 1005.22 & $\begin{array}{l}961.13,917.29,757.12,713.23 b \\
458.07\end{array}$ & & & [21] \\
\hline 106 & $\begin{array}{l}\text { Phenylpropanoid-derived } \\
\text { disaccharide esters }\end{array}$ & 24.2 & $220,290,315$ & 1181.4 & $\begin{array}{l}1133.38,1009.38,955.50 b, 809.41 \\
663.14\end{array}$ & & & \\
\hline 107 & $\begin{array}{l}\text { Phenylpropanoid-derived } \\
\text { disaccharide esters }\end{array}$ & 24.5 & $220,290,315$ & 1151.38 & $\begin{array}{l}1133.42,1103.35,1009.32,955.40 \mathrm{~b} \\
809.29\end{array}$ & & & {$[23]$} \\
\hline 108 & $\begin{array}{l}\text { Phenylpropanoid-derived } \\
\text { disaccharide esters }\end{array}$ & 24.6 & $220,290,315$ & 1151.4 & $\begin{array}{l}1133.38,1103.38,1009.33,955.39 b \\
809.29\end{array}$ & & & [23] \\
\hline 109 & Unknown & 24.7 & $220,280,315$ & 623.28 & $\begin{array}{l}591.21,551.26,486.13,460.17 \mathrm{~b} \\
352.16,297.07\end{array}$ & & & \\
\hline 110 & Tatariside $b^{*}$ & 25.0 & $220,290,315$ & 893.27 & $\begin{array}{l}851.24,747.22 b, 705.27,687.33 \\
569.19\end{array}$ & $\begin{array}{l}\text { 705.24b, 687.25, 663.22, } \\
569.16,527.18,322.96\end{array}$ & 146 & \\
\hline 111 & $\begin{array}{l}\text { Methyl derivative of Emodin } \\
\text { bianthrone-hexose-(malonic } \\
\text { acid)-hexose }\end{array}$ & 25.1 & 220,278 & 933.2 & $889.42 b, 727.19,685.20,416.06$ & & & [21] \\
\hline 112 & $\begin{array}{l}\text { Derivative of Emodin } \\
\text { bianthrone-di-hexose }\end{array}$ & 25.4 & 220,278 & 1019.24 & $\begin{array}{l}975.25,931.43 b, 889.25,727.20 \\
458.07\end{array}$ & & & [21] \\
\hline 113 & Vanicoside B (isomer) & 25.6 & $220,290,315$ & 955.37 & $809.26 b, 663.19$ & $663.26 \mathrm{~b}, 485.20,453.09$ & 146 & \\
\hline 114 & Unknown & 25.8 & $220,280,315$ & 801.29 & $\begin{array}{l}759.25 \mathrm{~b}, 741.50,655.19,613.25 \\
571.13,331.05\end{array}$ & $\begin{array}{l}\text { 613.18b, 595.13, 571.15, } \\
553.12,330.95\end{array}$ & 146 & \\
\hline 115 & Tatariside b (isomer) & 26.0 & $220,290,315$ & 893.28 & $\begin{array}{l}851.27,747.21 b, 705.29,687.31 \\
569.18\end{array}$ & $\begin{array}{l}705.26 \mathrm{~b}, 687.37,663.34, \\
569.23,527.31,322.98\end{array}$ & 146 & \\
\hline 116 & Vanicoside B * & 26.4 & $220,290,315$ & 955.29 & $\mathbf{8 0 9 . 2 2 b}, 663.20,453.05$ & $663.21 \mathrm{~b}, 485.20,323.05$ & 146 & \\
\hline 117 & Lapathoside a & 26.6 & $220,290,315$ & 985.3 & $839.24 b, 809.24,663.22,483.12$ & $663.20 \mathrm{~b}, 485.08,322.85$ & 176 & {$[16,23]$} \\
\hline
\end{tabular}


Table 1. Cont.

\begin{tabular}{|c|c|c|c|c|c|c|c|c|}
\hline Nr. & Identification & Rt & $\lambda \max (\mathrm{nm})$ & $m / z[\mathbf{M}-\mathbf{H}]^{-}$ & MS $^{2}$ ions & $\mathrm{MS}^{3}$ ions & NL Da & References \\
\hline 118 & $\begin{array}{l}\text { Diacetyl derivative of } \\
\text { (diacetoxy-methoxyphenyl) } \\
\text { acroyl-O-p-coumaroyl-O- } \\
\text { caffeoylquinic acid }\end{array}$ & 26.7 & $220,288,325$ & 861.3 & $\begin{array}{l}\text { 819.29b, 801.25, 777.25, 759.25, } \\
\text { 685.20, 643.17, 601.20, 583.18, } \\
\text { 559.25, 513.17, 361.01 }\end{array}$ & $643.19 b, 625.18,601.15,583.15$ & 176 & [18] \\
\hline 119 & Lapathoside b & 26.8 & $220,290,315$ & 1015.31 & $\begin{array}{l}869.23,839.23 b, 693.19,663.22 \\
483.15\end{array}$ & $\begin{array}{l}693.23,663.20 \mathrm{~b}, 645.28, \\
499.09,322.89\end{array}$ & 176 & [26] \\
\hline 120 & Questin* & 27.0 & $222,286,430$ & 282.94 & $267.89,239.85 \mathrm{~b}$ & & & \\
\hline 121 & $\begin{array}{l}\text { Phenylpropanoid-derived } \\
\text { disaccharide esters }\end{array}$ & 27.1 & $220,290,315$ & 1193.48 & $\begin{array}{l}1175.45,1145.50,1051.38,997.44 \mathrm{~b} \\
851.31,821.30\end{array}$ & & & \\
\hline 122 & $\begin{array}{l}\text { Phenylpropanoid-derived } \\
\text { disaccharide esters }\end{array}$ & 27.2 & $220,290,315$ & 1163.41 & $\begin{array}{l}\text { 1145.45b, 1133.51, 999.37, 955.30, } \\
851.15,809.28\end{array}$ & & & \\
\hline 123 & $\begin{array}{l}\text { Diacetyl derivative of } \\
\text { (diacetoxy-methoxyphenyl) } \\
\text { acroyl-O-p-coumaroyl-O- } \\
\text { caffeoylquinic acid }\end{array}$ & 27.3 & $220,288,325$ & 861.32 & $\begin{array}{l}819.29 \mathrm{~b}, 801.25,777.25,759.25 \\
\mathbf{6 8 5 . 2 0}, 643.17,601.20,583.18 \\
559.25,513.17,361.01\end{array}$ & $643.17 \mathrm{~b}, 625.18,601.15,583.15$ & 176 & {$[18]$} \\
\hline 124 & Vanicoside B (isomer) & 27.4 & $220,290,315$ & 955.28 & $809.20 b, 663.19,453.04$ & $663.23 b, 485.20,323.06$ & 146 & \\
\hline 125 & Dihydroferuloyl vanicoside B & 27.8 & $220,290,315$ & 1133.4 & $1115.49 b, 1103.65,997.32,969.37$ & & & {$[16,23]$} \\
\hline 126 & Unknown & 28.0 & $220,290,315$ & 1071.38 & $\begin{array}{l}\text { 1053.46b, 1041.64, 935.32, 907.40, } \\
866.38,717.11\end{array}$ & & & \\
\hline 127 & $\begin{array}{l}\text { Diacetyl derivative of } \\
\text { (diacetoxy-methoxyphenyl) } \\
\text { acroyl-O-p-coumaroyl-O- } \\
\text { caffeoylquinic acid }\end{array}$ & 28.1 & $220,288,325$ & 861.32 & $\begin{array}{l}\text { 819.29b, 801.25, 777.25, 759.25, } \\
\text { 685.20, 643.17, 601.20, 583.18, } \\
559.25,513.17,361.01\end{array}$ & $643.17 \mathrm{~b}, 625.18,601.15,583.15$ & 176 & [18] \\
\hline 128 & $\begin{array}{l}\text { Emodin bianthrone-hexose- } \\
\text { malonic acid }\end{array}$ & 28.2 & $220,278,350$ & 757.14 & $713.25 b, 509.10,458.12$ & & & {$[21]$} \\
\hline 129 & Dihydroferuloyl vanicoside B & 28.5 & $220,290,315$ & 1133.38 & $1115.49 \mathrm{~b}, 1103.50,997.33,969.38$ & & & {$[16,23]$} \\
\hline 130 & $\begin{array}{l}\text { Derivative of Emodin } \\
\text { bianthrone-di-hexose }\end{array}$ & 28.7 & 220,278 & 1019.24 & $\begin{array}{l}975.38,931.43 \mathrm{~b}, 889.25,727.20 \\
458.07\end{array}$ & & & {$[21]$} \\
\hline 131 & Vanicoside A * & 29.0 & $220,290,315$ & 997.31 & $\begin{array}{l}955.29,851.24 b, 821.28,705.21 \\
453.05\end{array}$ & $809.24,705.29 b, 663.48,527.22$ & 146 & \\
\hline 132 & Tatariside $C$ & 29.1 & $220,290,315$ & 935.27 & $\begin{array}{l}893.27,789.22 b, 747.32,705.29 \\
611.17,569.18\end{array}$ & $747.26 \mathrm{~b}, 705.23,611.26,569.22$ & 146 & {$[19,27]$} \\
\hline 133 & Hydropiperoside b & 29.2 & $220,290,315$ & 1027.3 & $\begin{array}{l}985.38,967.30,881.25 b, 851.23 \\
705.20,453.09\end{array}$ & $\begin{array}{l}809.19,705.20 \mathrm{~b}, 663.20, \\
527.08,453.06,322.96\end{array}$ & 176 & [28] \\
\hline 134 & $\begin{array}{l}\text { Derivative of } \\
\text { (diacetoxy-methoxyphenyl) } \\
\text { acroyl-O-p-coumaroyl-O- } \\
\text { caffeoylquinic acid }\end{array}$ & 29.4 & $220,285,325$ & 965.36 & $\begin{array}{l}923.31,819.26,789.29 b, 747.22 \\
643.21\end{array}$ & $\begin{array}{l}777.31 b, 643.08,611.15 \\
569.05,361.06\end{array}$ & 146 & [18] \\
\hline 135 & $\begin{array}{l}\text { Derivative of } \\
\text { (diacetoxy-methoxyphenyl) } \\
\text { acroyl-O-p-coumaroyl-O- } \\
\text { caffeoylquinic acid }\end{array}$ & 29.7 & $220,285,325$ & 995.37 & $\begin{array}{l}953.33,819.23 b, 777.25,759.13 \\
611.24\end{array}$ & $\begin{array}{l}777.23 \mathrm{~b}, 735.18,643.29 \\
611.16,569.18\end{array}$ & 176 & {$[18]$} \\
\hline
\end{tabular}


Table 1. Cont.

\begin{tabular}{|c|c|c|c|c|c|c|c|c|}
\hline Nr. & Identification & Rt & $\lambda \max (\mathrm{nm})$ & $m / z[\mathbf{M}-\mathbf{H}]^{-}$ & MS $^{2}$ ions & MS $^{3}$ ions & NL Da & References \\
\hline 136 & $\begin{array}{l}\text { Isomer vanicoside } \\
\mathrm{A} / \text { vanicoside } \mathrm{F}\end{array}$ & 29.9 & $220,290,315$ & 997.32 & $\begin{array}{l}955.29,851.24 b, 821.28,705.21 \\
453.06\end{array}$ & $\begin{array}{l}809.22,705.27 \mathrm{~b}, 663.31 \\
527.20,323.01\end{array}$ & 146 & \\
\hline 137 & $\begin{array}{l}\text { Phenylpropanoid-derived } \\
\text { disaccharide esters }\end{array}$ & 30.3 & $220,290,315$ & 1175.43 & $1157.52 b, 1145.61,1039.33,1011.37$ & & & \\
\hline 138 & Emodin bianthrone-hexose & 30.35 & $220,278,350$ & 671.17 & $653.18,509.09,416.08 b, 253.95$ & $491.01,253.88 \mathrm{~b}$ & 162 & [21] \\
\hline 139 & Unknown & 30.4 & $220,265,325$ & $324.99 b, 244.93$ & 244.88 & & & \\
\hline 140 & Unknown & 30.7 & $220,265,325$ & 1113.43 & $1095.45 b, 1083.45,977.29,949.33$ & & & \\
\hline 141 & $\begin{array}{l}\text { Phenylpropanoid-derived } \\
\text { disaccharide esters }\end{array}$ & 31.4 & $220,290,315$ & $954.33[\mathrm{M}-3 \mathrm{H}]^{3}$ & $881.20[\mathrm{M}-2 \mathrm{H}]^{2}, 809.20,779.22 \mathrm{~b}$ & $633.09 b, 486.99$ & 176 & {$[23]$} \\
\hline 142 & $\begin{array}{l}\text { Emodin bianthrone-hexose- } \\
\text { malonic acid }\end{array}$ & 31.5 & $220,278,350$ & 757.16 & $\begin{array}{l}713.25 \mathrm{~b}, 671.25,509.10,502.00 \\
458.12\end{array}$ & & & [21] \\
\hline 143 & Vanicoside E & 32.1 & $220,290,315$ & 1039.31 & $997.24,893.25 b, 747.30,453.05$ & $\begin{array}{l}851.27,747.28 b, 705.40 \\
569.24,304.91\end{array}$ & 146 & {$[27,28]$} \\
\hline 144 & $\begin{array}{l}\text { Emodin bianthrone-hexose- } \\
\text { malonic acid }\end{array}$ & 32.7 & $220,278,350$ & 757.16 & $\begin{array}{l}713.21 b, 671.19,509.11,502.00 \\
458.12\end{array}$ & & & [21] \\
\hline 145 & $\begin{array}{l}\text { Methyl derivative of Emodin } \\
\text { bianthrone-hexose }\end{array}$ & 33.0 & $220,278,350$ & 685.18 & $416.07 \mathrm{~b}, 253.92$ & & & [21] \\
\hline 146 & $\begin{array}{l}\text { Methyl derivative of Emodin } \\
\text { bianthrone-hexose }\end{array}$ & 34.0 & $220,278,350$ & 685.17 & $416.07 \mathrm{~b}, 253.92$ & & & [21] \\
\hline 147 & Emodin * & 34.2 & $\begin{array}{l}220,248,265, \\
288,430\end{array}$ & 268.89 & $240.81,224.93 b, 181.68$ & & & \\
\hline 148 & $\begin{array}{l}\text { Methyl derivative of Emodin } \\
\text { bianthrone-hexose- } \\
\text { malonic acid }\end{array}$ & 34.6 & $220,278,350$ & 771.14 & $727.22 \mathrm{~b}, 502.05,458.07$ & & & [21] \\
\hline 149 & Unknown & 35.0 & $220,278,350$ & 721.41 & $675.39 b, 397.10$ & & & \\
\hline 150 & $\begin{array}{l}\text { Methyl derivative of Emodin } \\
\text { bianthrone-hexose- } \\
\text { malonic acid }\end{array}$ & 35.2 & $220,278,350$ & 771.15 & $727.24 \mathrm{~b}, 502.05,458.07$ & & & [21] \\
\hline 151 & $\begin{array}{l}\text { Methyl derivative of Emodin } \\
\text { bianthrone-hexose- } \\
\text { malonic acid } \\
\text { Methyl derivative of Emodin }\end{array}$ & 35.6 & $220,278,350$ & 771.14 & $727.23 b, 502.04,458.08$ & & & [21] \\
\hline 152 & $\begin{array}{l}\text { bianthrone-hexose- } \\
\text { malonic acid }\end{array}$ & 36.3 & $220,278,350$ & 771.15 & $727.23 b, 502.04,458.08$ & & & [21] \\
\hline 153 & Unknown & 37.5 & $225,280,325$ & 647.37b, 1203.74 & $601.34 \mathrm{~b}, 341.1$ & & & \\
\hline 154 & Unknown & 37.9 & $225,280,325$ & 723.42 & $677.40,397.09$ & & & \\
\hline 155 & Unknown & 38.3 & $220,278,350$ & 369.18 & $\begin{array}{l}351.12,311.02,292.99 b, 210.79 \\
170.76\end{array}$ & & & \\
\hline 156 & Unknown & 38.4 & $225,280,325$ & 559.35 & $513.28 b, 277.15,252.98$ & & & \\
\hline 157 & Unknown & 39.4 & $225,280,325$ & 559.36 & $513.29 b, 277.16,253.01$ & & & \\
\hline 158 & Unknown & 40.7 & 225,275 & 649.39 & 603.37 & & & \\
\hline
\end{tabular}


Table 1. Cont.

\begin{tabular}{|c|c|c|c|c|c|c|c|c|}
\hline Nr. & Identification & $\mathbf{R t}$ & $\lambda \max (\mathrm{nm})$ & $m / z[\mathbf{M}-\mathbf{H}]^{-}$ & MS $^{2}$ ions & MS $^{3}$ ions & NL Da & References \\
\hline 159 & $\begin{array}{l}\text { Isovitexin/vitexin } \\
\text { diglucoside }\end{array}$ & 41.0 & 269,333 & 755.39 & $593.25,575.29 b, 477.06,431.21$ & $533.25,503.21,431.19 b, 413.28$ & 162 & {$[29,30]$} \\
\hline 160 & Unknown & 41.5 & $220,278,360$ & 725.45 & $679.43 b, 397.09$ & & & \\
\hline 161 & Emodin bianthrone & 41.6 & $220,278,360$ & 509.14 & $491.08,253.88 \mathrm{~b}$ & & & [21] \\
\hline 163 & Unknown & 42.7 & $225,280,325$ & 561.59 & $515.32 \mathrm{~b}, 279.20,253.00$ & & & \\
\hline 164 & Unknown & 42.8 & $225,280,325$ & 625.39 & 579.36 & & & \\
\hline 165 & Emodin bianthrone isomer & 43.6 & $220,278,360$ & 509.14 & $491.06,253.88 \mathrm{~b}$ & & & [21] \\
\hline 166 & Unknown & 44.7 & $225,280,325$ & 651.41 & 605.4 & & & \\
\hline 167 & Unknown & 45.2 & $220,278,350$ & 757.4 & $\begin{array}{l}\text { 595.30, } 577.30,477.05 b, 433.22 \\
279.16\end{array}$ & $\begin{array}{l}535.27,505.24,475.23 \\
433.22 \mathrm{~b}, 279.13\end{array}$ & 162 & \\
\hline 168 & Unknown & 47.2 & $225,280,325$ & 563.39 & $517.34 \mathrm{~b}, 281.21,253.00$ & & & \\
\hline 169 & $\begin{array}{l}\text { Methyl derivative of } \\
\text { emodin bianthrone }\end{array}$ & 50.4 & $220,278,360$ & 523.18 & 253.89 & & & [21] \\
\hline 170 & $\begin{array}{l}\text { Alpha- } \\
\text { carboxyethylhydroxychroman }\end{array}$ & 54.4 & 292 & 277.19 & $259.13,233.06 b$ & & & [31] \\
\hline 171 & Unknown & 57.4 & $220,278,350$ & 279.2 & $261.11 b, 233.17$ & & & \\
\hline
\end{tabular}

b-base peak (the most abundant ion in the recorded spectrum), in bold-ions subjected to MS/MS fragmentation (if it's not obvious), *-isolated and/or characterised in our previous paper [5], ND-not determined. 


\subsubsection{Stilbenoids}

Almost all identified stilbenes with characteristic UV spectra with maxima about $\lambda_{\max } 220$, 305, $320 \mathrm{~nm}$ have been previously observed in studied materials [5]. No stilbenes were detected in R. sachalinensis. Compounds 20 (piceatannol glucoside) 22 (resveratroloside), 27 (piceid) and 45 (resveratrol), were characterized by HPLC-DAD-ESI-HR-TOF-MS and described in previous article [5]. Only compound 42 with deprotonated molecule at $m / z 389[\mathrm{M}-\mathrm{H}]^{-}$and fragmentation ion at $m / z$ 227 characteristic for resveratrol hexoside was noticed for the first time. Compound 42 was observed in small amount in $R$. japonica and $R x$. bohemica dichloromethane or diethyl ether fractions.

\subsubsection{Carbohydrates}

Deconvolution of an LC/MS mass chromatogram by using the Bruker's Dissect algorithm allowed to observed several carbohydrates in very similar retention times. Furthermore, the hydrophilic character of compounds and the lack of chromophores confirmed the presence of carbohydrates. Based on deprotonated molecule and fragmentation ions, compounds 1,2,3 and 4 were described as unknown carbohydrates (Table 1) [10]. Compound 1 exhibited deprotonated molecule at $m / z 341$ $[\mathrm{M}-\mathrm{H}]^{-}$, characteristic for dissacharids e.g., sucrose, which was confirmed by the fragmentation ions at $m / z 179$ [M - H-162 $]^{-}, 161$ and 143 characteristic for fructose. Compounds 2, 3 and 4 were more complex but contained the same fragmentation ions at $m / z 341$ and 179. More accurate analyses with using different method are needed to identify carbohydrates fully [32]. All apparent carbohydrates were observed in studied Reynoutria species water fractions.

\subsubsection{Flavan-3-ols and Procyanidins}

B-type procyanidins have different fragmentation patterns than A-type procyanidins and it was used to differentiate procyanidins by the type of linkages between monomeric units [12]. In studied material, there were observed only B-type procyanidins. Identified compounds possessed the same UV spectra characteristic for flavan-3-ols with maxima about $\lambda_{\max } 225,280$ and characteristic fragmentation patterns presented in the Figure 4.

Compound 11 was identified as catechin (deprotonated molecule at $m / z 289[\mathrm{M}-\mathrm{H}]^{-}$). Compound 16, an isomer of 11 with the same molecular mass was identified as epicatechin, both reported earlier in studied species [5] and confirmed with standards. Compounds 7, 9, 13, 33 with a deprotonated molecule at $m / z 577[\mathrm{M}-\mathrm{H}]^{-}$were identified as procyanidin dimers type $\mathrm{B}$ and compounds 10, 15, 19, 21 with deprotonated molecules at $m / z 865[\mathrm{M}-\mathrm{H}]^{-}$as procyanidin trimers type B [11-13].

Compound 25 with deprotonated molecule at $m / z 1153[\mathrm{M}-\mathrm{H}]^{-}$and with the main product ion at $m / z 865$ [M - H-288] ${ }^{-}$corresponding to procyanidin trimer type B was assigned to procyanidin tetramer type B. Procyanidins with high degree of polymerization, due to the mass range limitations of MS detector were identified by multiple charged ions. Compounds $\mathbf{1 4}$ and 26 possessed doublecharged ion with signals at $m / z 720[\mathrm{M}-2 \mathrm{H}]^{2-}$ and compound 31, double-charged ion with signals at $m / z 1008[\mathrm{M}-2 \mathrm{H}]^{2-}$. Taking into account derivative ions (Table 1 ), characteristic for fragmentation patterns of pentamer and heptamer $[12,14,15]$ they were tentatively assignment to procyanidin pentamers and procyanidin heptamer respectively. According to the literature, compounds 34 and 36 with signals at $m / z 1152[\mathrm{M}-2 \mathrm{H}]^{2-}$ were tentatively assignment to procyanidin octamers [15].

Procyanidin gallates were distinguished by their characteristic fragment ion spectra yielding losses of galloyl moiety (-152 Da). Based on the literature [11,13,15] compounds 17, 24, 43 were identified as procyanidin dimer monogallates with deprotonated molecule at $m / z 729[\mathrm{M}-\mathrm{H}]^{-}$, and peaks $\mathbf{1 8}, \mathbf{2 3}, \mathbf{3 0}, \mathbf{3 5}, 40$ as procyanidin trimer monogallates with deprotonated molecule at $m / z$ $1017[\mathrm{M}-\mathrm{H}]^{-}$. 


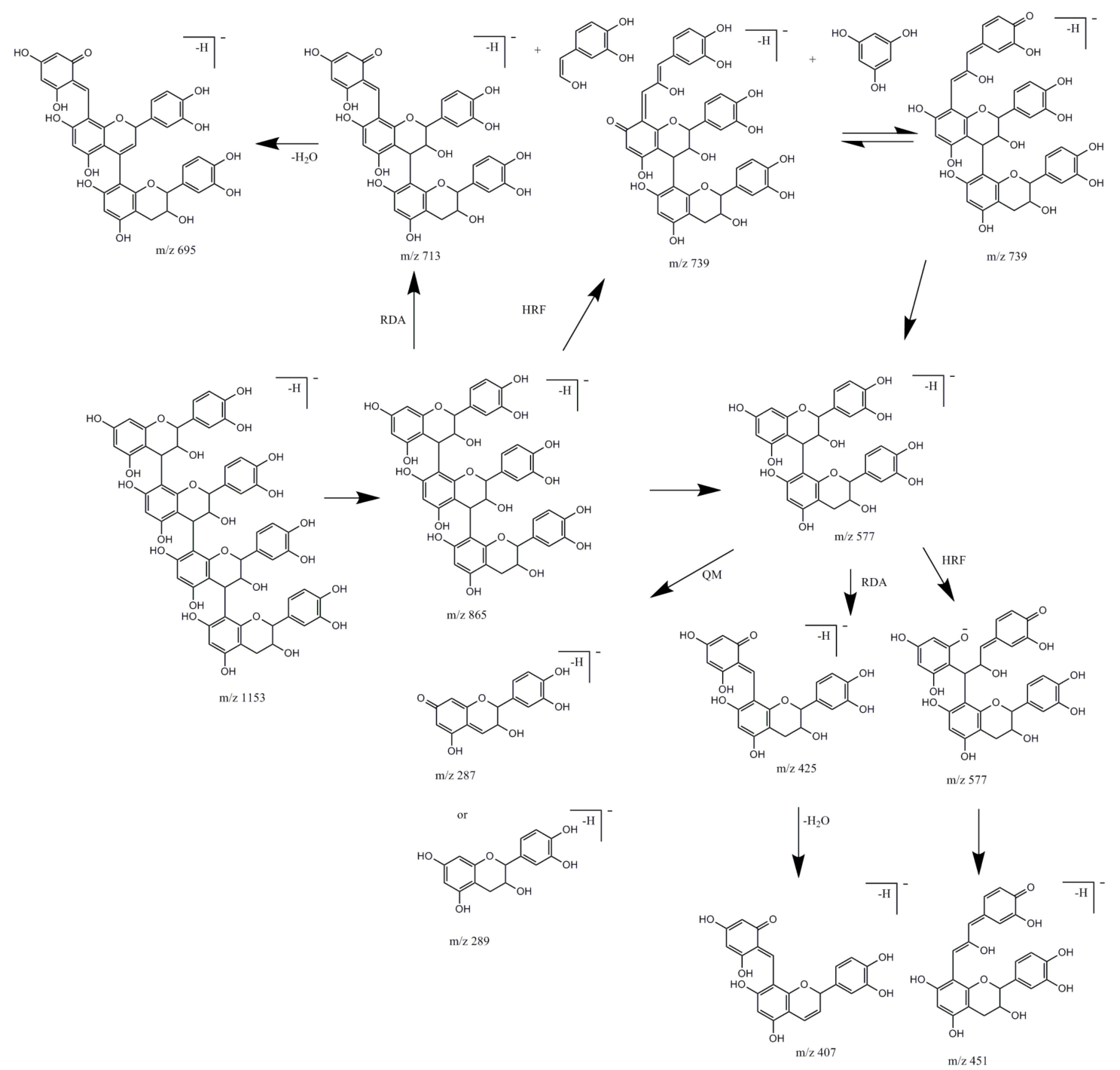

Figure 4. Fragmentation pathways of procyanidins in negative ion mode. RDA, retroDiels-Adler fragmentation; $\mathrm{HRF}$, heterocyclic ring fission; $\mathrm{QM}$ - quinone methide cleavage.

Compound 37 with a deprotonated molecule at $m / z 1305[\mathrm{M}-\mathrm{H}]^{-}$and a double- charged ion at $m / z 652[\mathrm{M}-2 \mathrm{H}]^{2-}$ as well as with fragmentation patterns characteristic for procyanidins, was tentatively assigned to procyanidin tetramer monogallate [15]. Compounds 29 and $\mathbf{4 6}$ revealed deprotonated molecules at $m / z 881[\mathrm{M}-\mathrm{H}]^{-}$and had characteristic fragmentation pattern for a procyanidin dimer digallate type B. Compound 28 with a deprotonated molecule at $m / z 1169$ $[\mathrm{M}-\mathrm{H}]^{-}$and a characteristic fragmentation pattern was tentatively assigned to procyanidin trimer digallate [13]. Compound 39 possessed triple-charged ions with signals at $m / z 796$ and fragmentation ions characteristic for procyanidin gallate like $m / z 1305$ [tetramer gallate] and others (Table 1). It was assigned as procyanidin gallate; probably it is built with more than five monomers and one or more galloyl groups. Similar compound $\mathbf{4 1}$ assigned as procyanidin gallate, in their fragmentation possessed ions characteristic for procyanidin gallate, like $m / z 881$ for dimer digallate, $m / z 1151$ [15] for tetramer procyanidin type A [32], $m / z 441$ for catechin monogallate [13] and others (Table 1). Compound 32 with deprotonated molecule at $m / z 441[\mathrm{M}-\mathrm{H}]^{-}$and confirmed with standards was identified as epicatechin-3-O-gallate.

Procyanidins with degree of polymerization higher than dimers were described for the first time in $R$. $\mathrm{x}$ bohemica. Whereas most of them were earlier observed in extract of rhizome of $R$. japonica by analyzed it on HPTLC-MS [15]. Using different analytical methods-HPLC-DAD-MS, we confirmed the 
presence of high order procyanidins, up to decamers in the rhizomes of $R$. japonica and brought new data on the presence of these compounds in other Reynoutria species. The presence of a procyanidin trimer digallate has not been reported from any of the studied species before.

\subsubsection{Anthraquinones}

Compounds 44 and 60 has been previously reported in studied species by using HR-MS analysis [5] and identified as emodin glucoside. Compounds $\mathbf{4 4}$ and $\mathbf{6 0}$ showed the most abundant product ions at $m / z 269$ [M $-\mathrm{H}-162]^{-}$(due to loss of a glucosyl moiety) which was characteristic for emodin. It is supposed that peak 44 corresponds to emodin-1-O-glucoside and peak 60 to emodin-8-O-glucoside. Compounds 78, 120 and 147 were also characterized earlier using a high-resolution time-of-flight MS [5]. Here, the deprotonated molecule at $\mathrm{m} / \mathrm{z} 517[\mathrm{M}-\mathrm{H}]^{-}$for compound 78 showed the most abundant product ion at $m / z 473[\mathrm{M}-\mathrm{H}-44]^{-}$and product ion at $\mathrm{m} / \mathrm{z}$ 431 [M-H-44-42] $]^{-}$what correspond to fragmentation pattern of emodin-8-O-(6'-O-malonyl)-glucoside, earlier identified in rhizome of $R$. japonica [11]. In our study, compound 78 was observed also in $R$. x bohemica. Compound 120 with a deprotonated molecule at $m / z 283[\mathrm{M}-\mathrm{H}]^{-}$showed the most abundant product ion at $\mathrm{m} / \mathrm{z} 240$ and a product ion at $\mathrm{m} / \mathrm{z} 268$ that correspond to the fragmentation pattern of questin [11]. Questin was observed in all extracts, but only in small amounts in $R$. sachalinensis. The next antraquinone identified in all extracts was emodine (compound 147), due to its characteristic UV spectrum and fragmentation (a most abundant product ion at $m / z 225$ and smaller product ions at $m / z 241$ and 182).

Compound 73 with a deprotonated molecule at $m / z 511[\mathrm{M}-\mathrm{H}]^{-}$a consecutive loss of $\mathrm{SO}_{3}$ (the fragmentation ion at $m / z 431[\mathrm{M}-\mathrm{H}-80]^{-}$) and a glucoside $m / z 269[\mathrm{M}-\mathrm{H}-80-162]^{-}$ led to the formation of an aglycone ion identified as emodin, proved by the diagnostic ions $m / z 241$ and 225. Based on the literature [11,20] compound 73 was tentatively identified as emodin-O-(sulfonyl)-glucoside observed only in the R. japonica butanol fraction.

Compounds 66, 92 and 96 were observed in studied species for the first time, all of them exhibited the same deprotonated molecule at $m / z 919[\mathrm{M}-\mathrm{H}]^{-}$and similar fragment ions despite little difference in intensity. The proposed fragmentation map is shown in Figure 5.

Malonyl-substituted type glucosides are widely found in Fallopia multiflora and Rheum plants. Due to a lack of standard compounds and the fact no dianthrones have been found earlier in $R$. japonica, $R$. x bohemica and $R$. sachalinensis, the structural characterization of the new dianthrone glycosides was referred to the literature on Rheum genus plants and F. multiflora [21,33,34] in which the MS fragmentation behavior of dianthrone glycosides was well described. Malonyl-substitution of dianthrone glycosides was earlier described in F. multiflora [21]. In our study, compounds 66, 92 and 96 tentatively assigned as emodin bianthrone-hexose-(malonic acid)-hexose were observed only in $R$. $\mathrm{x}$ bohemica extract and $R$. x bohemica butanol fraction.

Compounds 84, 102, 111 exhibited the same deprotonated molecule at $m / z 933[\mathrm{M}-\mathrm{H}]^{-}$and the same most abundant fragment ion at $m / z 889[\mathrm{M}-\mathrm{H}-44]^{-}$due to loss of $\mathrm{CO}_{2}$ and fragment ion at $m / z 727$ [M - H-44-162] $]^{-}$produced by a cleavage of a glucosidic bond. The deprotonated molecule at $m / z 933[\mathrm{M}-\mathrm{H}]^{-}$differed from those of compounds 66, 92,96 by $14 \mathrm{Da}$, which corresponds to a methyl moiety.

Based on their fragmentation patterns (similar to that presented in Figure 5, but with the addition of a methyl moiety) and literature, these compounds were tentatively identified as methyl derivatives of emodin bianthrone-hexose-(malonic acid)-hexose [21] which were observed as small peaks only in R. $\times$ bohemica butanol fraction. Compounds 89, 112, 130 with the same deprotonated molecules at $\mathrm{m} / \mathrm{z}$ $1019[\mathrm{M}-\mathrm{H}]^{-}$and fragmentation ions like described above: $m / z 889,727,458$, suggest the presence of methyl derivatives of emodin bianthrone-hexose-(acetyl)-hexose. The mentioned compounds are fragmented to ions at $m / z 975[\mathrm{M}-\mathrm{H}-44]^{-}$due to loss of $\mathrm{CO}_{2}$, the most abundant product ion is at $\mathrm{m} / \mathrm{z}$ $931[\mathrm{M}-\mathrm{H}-44 \times 2]^{-}$due to the loss of a second $\mathrm{CO}_{2}$. However, because of the many possible structures of compounds $89, \mathbf{1 1 2}, \mathbf{1 3 0}$, they were described as derivatives of emodin bianthrone-di-hexose. 


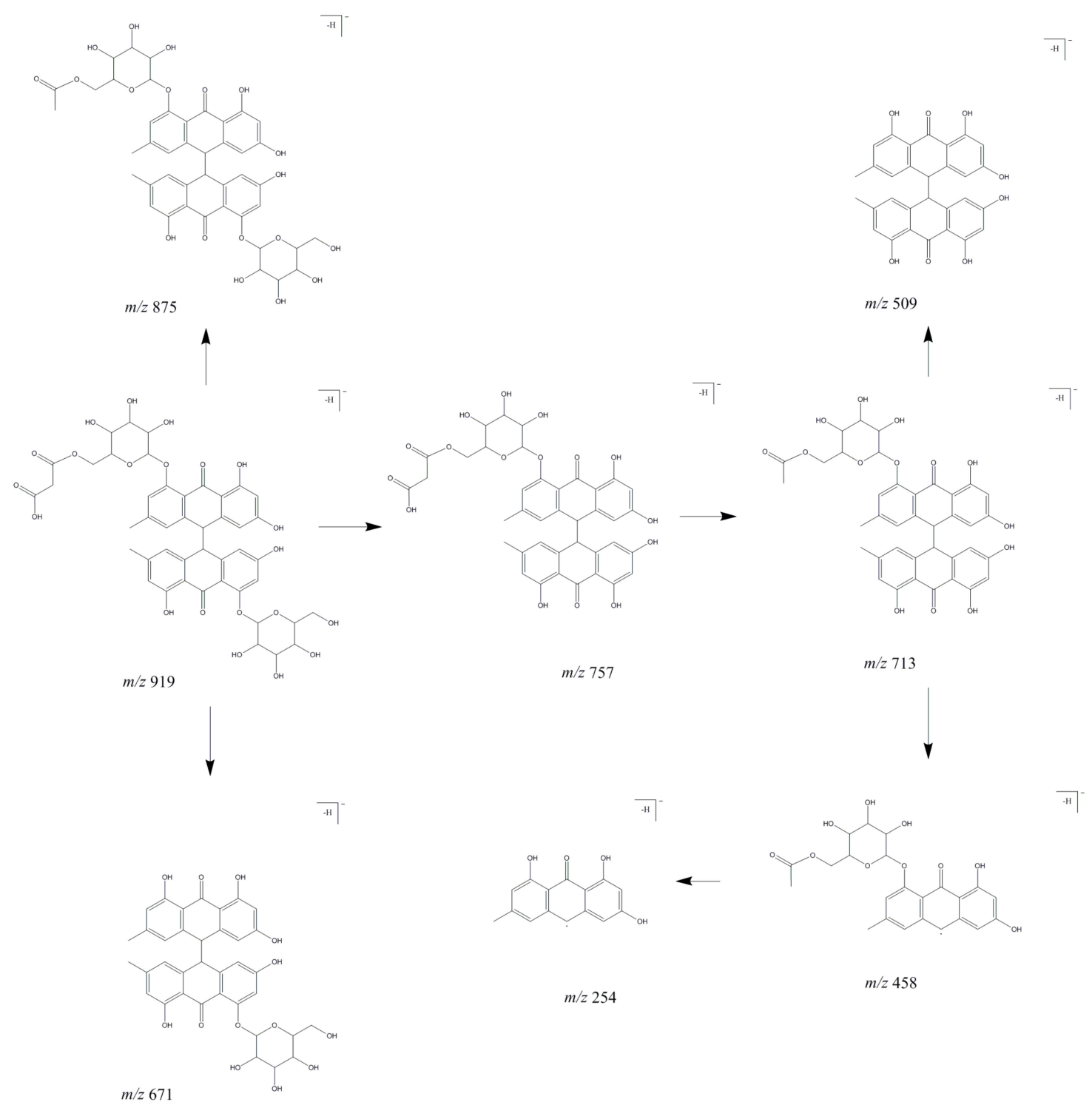

Figure 5. Proposed fragmentation pathway for peaks 66, 92, and 96.

The exact structure of these compounds requires detailed research. Compounds 89, 112, 130 were observed only in R. x bohemica extract and butanol fraction. Compounds 128, 142, 144 showed the same deprotonated molecule at $m / z 757[\mathrm{M}-\mathrm{H}]^{-}$. Due to the characteristic fragmentation ions at $m / z 713,509,458$ (Figure 5), they were tentatively identified as emodin bianthrone-hexose-malonic acids. These compounds were mainly observed in $R$. $\mathrm{x}$ bohemica extract and fractions and as a small peak in the $R$. japonica diethyl ether fraction. None of them were observed in $R$. sachalinensis.

Compounds 148, 150, 151, 152 exhibited the same deprotonated molecule at $m / z 771[\mathrm{M}-\mathrm{H}]^{-}$, that differed from peaks described above for $128,142,144$ by $14 \mathrm{Da}$, what could correspond to a methyl moiety loss. The most abundant product ion at $m / z 727[\mathrm{M}-\mathrm{H}-44]^{-}$was observed due to the loss of $\mathrm{CO}_{2}$. The product ion at $m / z 502[\mathrm{M}-\mathrm{H}-269]^{-}$was caused by the 10-10' homolytic cleavage of anthrone and the product ion at $m / z 458$ [M - H-44-269 $]^{-}$by cleavage of anthrone and loss of $\mathrm{CO}_{2}$. Peaks were tentatively identified as a methyl derivative of emodin bianthrone-hexose-malonic acid. Peaks were observed only in $R$. $\mathrm{x}$ bohemica extract and fractions.

Compounds 67 and 105 showed the same deprotonated molecule at $m / z 1005[\mathrm{M}-\mathrm{H}]^{-}$. Fragmentation ion at $m / z 757$ [M - H-248] $]^{-}$could represent emodin bianthrone-hexose-malonic acid as confirmed by subsequent fragmentation ions: the most abundant ion at $m / z 713$ [M $-\mathrm{H}-248-44]^{-}$ due to the loss of $\mathrm{CO}_{2}$, product ion at at $m / z 458$ [M $\left.-\mathrm{H}-248-44-255\right]^{-}$by cleavage of anthrone 
(Figure 5). Moreover, a deprotonated molecule at $m / z 1005[\mathrm{M}-\mathrm{H}]^{-}$after loss of $\mathrm{CO}_{2}$ created an ion at $m / z 961$ [M - H-44] $]^{-}$and after more loss of $\mathrm{CO}_{2}$ an ion at $m / z 917$ [M $\left.-\mathrm{H}-44 \times 2\right]^{-}$was created. Due to the many possible structures of compounds 67 and 105, they were described as derivative of emodin bianthrone-hexose-malonic acid. The exact structure of these compounds requires more detailed research. Compounds were observed in the butanol fractions of all studied Reynoutria species.

Compound 138 observed in extract of $R$. japonica and $R$. x bohemica exhibited a deprotonated molecule at $m / z 671[\mathrm{M}-\mathrm{H}]^{-}$and a product ion at $m / z 653[\mathrm{M}-\mathrm{H}-18]^{-}$, due to the loss of $\mathrm{H}_{2} \mathrm{O}$ moiety, a product ion at $m / z 509[\mathrm{M}-\mathrm{H}-162]^{-}$by loss of a hexosyl moiety, and the most abundant product ion at $m / z 416[\mathrm{M}-\mathrm{H}-255]^{-}$caused by the $10-10^{\prime}$ homolytic cleavage of anthrone and the product ion at $m / z 254$ [M - H-255-162]- by cleavage of anthrone and hexosyl moieties (Figure 5). Based on fragmentation pattern and literature, compound 138 was tentatively identified as emodin bianthrone-hexose [21]. Compounds 145 and 146 with the same deprotonated molecules at $m / z 685$ $[\mathrm{M}-\mathrm{H}]^{-}$differed from peak 138 by $14 \mathrm{Da}$ what corresponds to loss of a methyl moiety. What is more, compounds 145 and 146 exhibited product ions at $m / z 416$ and 254, described above. These compounds were tentatively identified as methyl derivatives of emodin bianthrone-hexose. The compounds were observed only in $R$. x bohemica dichloromethane and diethyl ether fractions.

Compounds 161 and 165 with the same deprotonated molecules at $m / z 509[\mathrm{M}-\mathrm{H}]^{-}$, fragmentation ions at $m / z 491[\mathrm{M}-\mathrm{H}-18]^{-}$, due to the loss of $\mathrm{H}_{2} \mathrm{O}$ and fragmentation ions at $m / z 254$ [M - H-255] ${ }^{-}$caused by the 10-10' homolytic cleavage of anthrone were tentatively identified as emodin bianthrones (Figure 5). The compounds were observed in $R$. japonica and in $R . \mathrm{x}$ bohemica fractions.

Compound 169 exhibited the same UV spectra with a maximum about $\lambda_{\max } 220,278,360 \mathrm{~nm}$, like compounds 161 and 165. Deprotonated molecule at $m / z 523[\mathrm{M}-\mathrm{H}]^{-}$fragmented to ion at $m / z$ 254 [M - H-269] ${ }^{-}$caused by the 10-10' homolytic cleavage of anthrone. Compound 169 differed from peak 161 and 165 by 14 Da that corresponds to a methyl moiety. The compound was tentatively identified as methyl derivative of emodin bianthrone.

\subsubsection{Phenylpropanoid Disaccharide Esters}

Phenylpropanoid-derived disaccharide esters possess a sucrose core carrying a varying number of O-substituents, including phenylpropanoid, acetyl, benzoyl, $p$-methoxybenzoyl, and $p$-hydroxy-benzoyl groups. Peaks 48, 74, 76, 87, 101, 88, 100, $103,110,115,113,116,124,117,125,129,131,136$ corresponding to acethyl lapathoside D, lapathoside C and isomers, e.g., hydropiperoside A [28], hydropiperoside and isomer, (3,6-O-di- $p$-coumaroyl)- $\beta$-fructofuranosyl-( $2 \rightarrow 1)-\left(2^{\prime}\right.$-O-acetyl-6'-O-feruloyl)- $\beta$-glucopyranoside and an isomer, vanicoside $C$, tatariside $B$ and an isomer, vanicoside $B$ and isomers, lapathoside $A$, dihydroferuloyl vanicoside $B$ and an isomer, vanicoside $A$ and an isomer (Table 1) were observed in the studied species previously $[5,16,23]$. The remaining phenylpropanoid disaccharide esters were detected in the present study for the first time. The identified phenylpropanoid-derived disaccharide esters possessed the same UV-Vis spectra characteristic for flavan-3-ols with maxima at about $\lambda_{\max }=220,290,315 \mathrm{~nm}$. Compounds 62,63 and 80 possessed the same deprotonated molecules at $m / z 717[\mathrm{M}-\mathrm{H}]^{-}$and similar fragmentation patterns with the most abundant ions at $m / z 571$ $[\mathrm{M}-\mathrm{H}-146]^{-}$caused by a loss of deoxyhexosyl, which gives in the $\mathrm{MS}^{3}$ analysis similar ions with the most abundant one at $m / z 529$ [M $-\mathrm{H}-42]^{-}$produced by loss of acetyl. According to [19] these compounds were tentatively identified as tatariside $\mathrm{E}$ and isomers. Compounds 83 and 95 with deprotonated molecules at $m / z 759[\mathrm{M}-\mathrm{H}]^{-}$and with characteristic fragmentation patterns (Table 1), were tentatively assigned as tatariside A and an isomer [19]. Both tatariside E and tatariside A were previously isolated from Fagopyrum tataricum [19]. Compound 119 observed in the diethyl ether fraction of $R$. sachalinensis and $R$. x bohemica with a deprotonated molecule at $m / z 1015[\mathrm{M}-\mathrm{H}]^{-}$and characteristic fragments was assigned as lapathoside $\mathrm{B}$, isolated and described earlier from Polygonum lapathifolium [26]. Compound 132 with a deprotonated molecule at $m / z 935[\mathrm{M}-\mathrm{H}]^{-}$was identified 
as tatariside C, earlier isolated from Fagopyrum tataricum [19,27]. Compound 132 had an additional acetyl group relative to tatariside B. Fragmentation ions of compound $\mathbf{1 3 2}$ were characteristic for tatariside B, e.g., $m / z 893$ and others (Table 1). Compound 133, observed in all studied species, with a deprotonated molecule at $m / z 1027[\mathrm{M}-\mathrm{H}]^{-}$was tentatively identified as hydropiperoside $\mathrm{B}$, isolated for the first time from Polygonum hydropiper [28]. The deprotonated molecule at $m / z 1027$ gave a product ion at $m / z 985$, which corresponds to the loss of the acetyl group from hydropiperoside $\mathrm{B}$ and was the same as the deprotonated molecule of lapathoside A. Similar compound 143 with a deprotonated molecule at $m / z 1039[\mathrm{M}-\mathrm{H}]^{-}$was identified as vanicoside $\mathrm{E}$, after losing the acetyl group, giving a product fragmentation ion at $m / z 997[\mathrm{M}-\mathrm{H}-42]^{-}$, characteristic for the deprotonated vanicoside A molecule [28]. Vanicoside E was observed in the diethyl ether fraction of $R$. sachalinensis and in small amounts in R. x bohemica. Compounds 107 and 108 with deprotonated molecules at $m / z 1151[\mathrm{M}-\mathrm{H}]^{-}$were earlier observed in rhizomes of $R$. sachalinensis [23] and were described as undefined phenylpropanoid glucoside. Compounds 107, 108 gave fragmentation ions characteristic for dihydroferuloyl vanicoside $\mathrm{B}$ at $m / z 1133$ and for vanicoside $\mathrm{B}, \mathrm{m} / z$ 955, $m / z$ 809, and were observed in all studied species. Compound 106 with a deprotonated molecule at $m / z 1181[\mathrm{M}-\mathrm{H}]^{-}$, observed in small amounts only in the diethyl ether fraction of $R$. sachalinensis and $R$. x bohemica, was noticed there for the first time. It has been described as a disaccharide phenylpropanoid ester derivative due to its UV-Vis spectrum and fragmentation ions, characteristic for this group of compounds (Table 1). Compounds 121, 122 and 137 with deprotonated molecules at $m / z$ 1193, 1163 and 1175, respectively, were observed for the first time in the studied species and were described as disaccharide ester derivatives of phenylpropanoids due to fragmentation ions such as $m / z 997$ (vanicoside A), $m / z 955$ (vanicoside B). Compound 141 which was observed only in the ethyl acetate fraction of $R$. sachalinensis, possessed a triple-charged ion with a signal at $m / z 954$, but also fragmentation ions at $m / z 809$ characteristic of lapathoside $C, m / z 779$ characteristic of hydropiperoside, as well an UV-Vis spectrum with maxima at $\lambda_{\max } 220,290,315 \mathrm{~nm}$ and this compound was described as a disaccharide ester derivative of phenylpropanoid. Compound $\mathbf{5 0}$ observed in R.sachalinensis and $R$. $\mathrm{x}$ bohemica fractions was tentatively assigned as a disaccharide ester phenylpropanoid derivative because of its fragmentation ions at $m / z 613,571$, similar to the fragmentation ions of tatariside A (compound 83). Compound 94 was tentatively assigned as a phenylpropanoid disaccharide ester derivative because of its UV-Vis spectrum similarity and fragmentation ions at $\mathrm{m} / \mathrm{z} 851$ ((3,6-O-di- $p$-coumaroyl)- $\beta$-fructofuranosyl- $(2 \rightarrow 1)-\left(2^{\prime}\right.$-O-acetyl-6'-O-feruloyl)- $\beta$-glucopyranoside) and ions at $m / z 633,453$ similar to the fragmentation ions of hydropiperoside.

\subsubsection{Lignin Oligomers}

Compounds tentatively identified as lignin oligomers (LOs) were observed in the dichloromethane fractions of studied Reynoutria species. All LOs were seen in the studied raw materials for the first time. Identification was made based on the fragmentation pattern of the LOs and the UV/VIS spectrum and comparisons with the literature.

Coniferyl alcohol (G unit), sinapyl alcohol (S unit) and p-coumaryl alcohol (H unit) are linked covalently, forming ether, ester and carbon-carbon bonds, which repeat to provide the great complexity of lignin [25]. The degree of polymerization in natural lignin is difficult to measure because it is supposed that it fragments during extraction [35]. Therefore, lignin fragments, oligomers of lignin, are the species most often identified in plant extracts. Compounds $\mathbf{7 1}$ and $\mathbf{7 2}$ were observed only in the dichloromethane fraction of $R$. sachalinensis and were identified based on fragmentation patterns described in [25]. The deprotonated molecule of compound 71 at $m / z 643[\mathrm{M}-\mathrm{H}]^{-}$was tentatively identified as a trimer of lignin $\beta-O-4$-linked $S$ unit with syringaresinol $\left[S-\left(\beta-O-4^{\prime}\right)-S-\left(\beta-\beta^{\prime}\right)-S\right]$ due to its fragmentation pattern which corresponds to that described by Evtuguin et al. [22]. The characteristic and most abundant fragmentation ion at $\mathrm{m} / \mathrm{z} 417$ corresponds to deprotonated syringaresinol (Figure 6). 


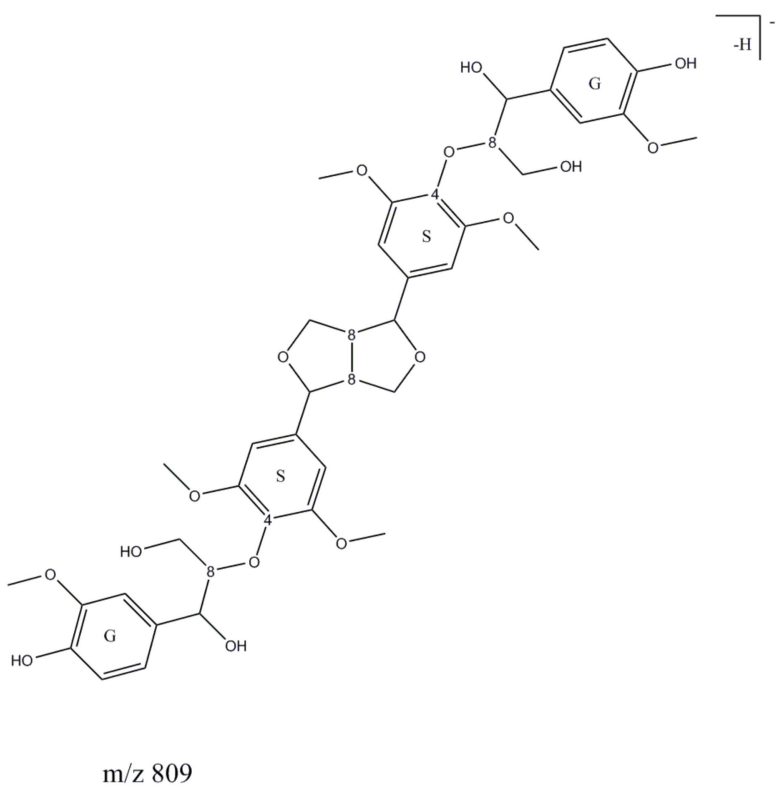

G(8-O-4)S(8-8)S(8-O-4)G

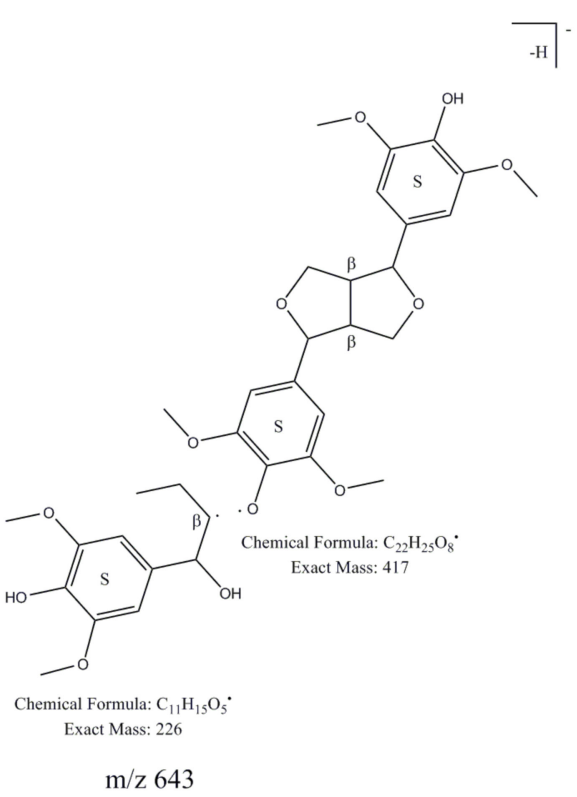

S- $\left(\beta-\mathrm{O}-4^{\prime}\right)-\mathrm{S}-\left(\beta-\beta^{\prime}\right)-\mathrm{S}$

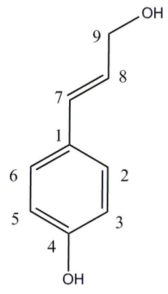

p-coumaryl alcohol $\mathrm{H}$ unit

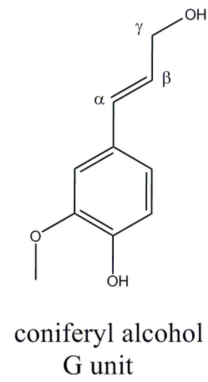

G unit

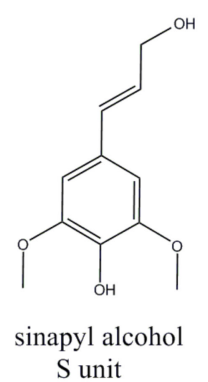

Figure 6. Lignin oligomers.

Compound 72 with a deprotonated molecule at $m / z 869$ differs by 226 Da from compound 71, what correspond to the syringyl phenylpropane unit. Based on the fragmentation pattern, which was similar to that of peak 71 and based on [22], peak 72 was assigned as tetramer lignin, $S-\left(8-O-4^{\prime}\right)-S-\left(8-O-4^{\prime}\right)-S-\left(8-8^{\prime}\right)-S$. Compound 81, observed as very small peak in the dichloromethane fraction of $R$. sachalinensis was described as a derivative of lignin-S(8-8)S. UV/VIS spectrum $\left(\lambda_{\max }\right.$ at 220 and $280 \mathrm{~nm})$ and its fragmentation ions at $\mathrm{m} / \mathrm{z} 417$ and $387\left(-\mathrm{CH}_{2} \mathrm{O}\right)$, suggest that compound 81 is composed of syringaresinol. Compounds 79, 85 and 93 with the same deprotonated molecules at $m / z 809[\mathrm{M}-\mathrm{H}]^{-}$and fragmentation ions were observed in all dichloromethane fractions of Reynoutria species. The deprotonated molecule at $m / z 809[\mathrm{M}-\mathrm{H}]^{-}$suggested a tetrameric compound structure, composed of two $\mathbf{G}$ and two $\mathbf{S}$ units (Figure 6). MS/MS spectral peaks at $m / z 791\left(-\mathrm{H}_{2} \mathrm{O}\right)$, $773\left(-2 \mathrm{H}_{2} \mathrm{O}\right), 761\left(-\mathrm{CH}_{2} \mathrm{O}\right.$ and $\left.\mathrm{H}_{2} \mathrm{O}\right), 743\left(-\mathrm{CH}_{2} \mathrm{O}\right.$ and $\left.2 \mathrm{H}_{2} \mathrm{O}\right)$ indicated the presence of two $\beta$-aryl ether units and a fragmentation ion at $m / z 417$ corresponds to deprotonated syringaresinol [24]. This MS and MS/MS spectrum was similar to the spectrum of oligolignol: G(8-O-4)S(8-8)S(8-O-4)G [24] called hedyotisol [36].

\subsubsection{Other Hydroxycinnamic Acid Derivatives}

The deprotonated molecule at $m / z 735[\mathrm{M}-\mathrm{H}]^{-}$for compound $\mathbf{6 1}$ was observed in all extracts. The peak showed a product ion at $m / z 693[\mathrm{M}-\mathrm{H}-42]^{-}$, due to the loss of an acetyl moiety. The most abundant product ion at $m / z 559$ was due to the loss of a feruloyl or isoferuloyl group. The fragmentation pattern showed ions at $m / z 499$ and 337, which were characterized as a 
p-coumarylquinic acid moiety. Based on fragmentation pattern and comparisons with the literature, compound $\mathbf{6 1}$ was tentatively assigned as dihydroksyferuloyl-O-acetoxy- $p$-coumaroyl-O-caffeoylquinic acid [18] (Figure 7).
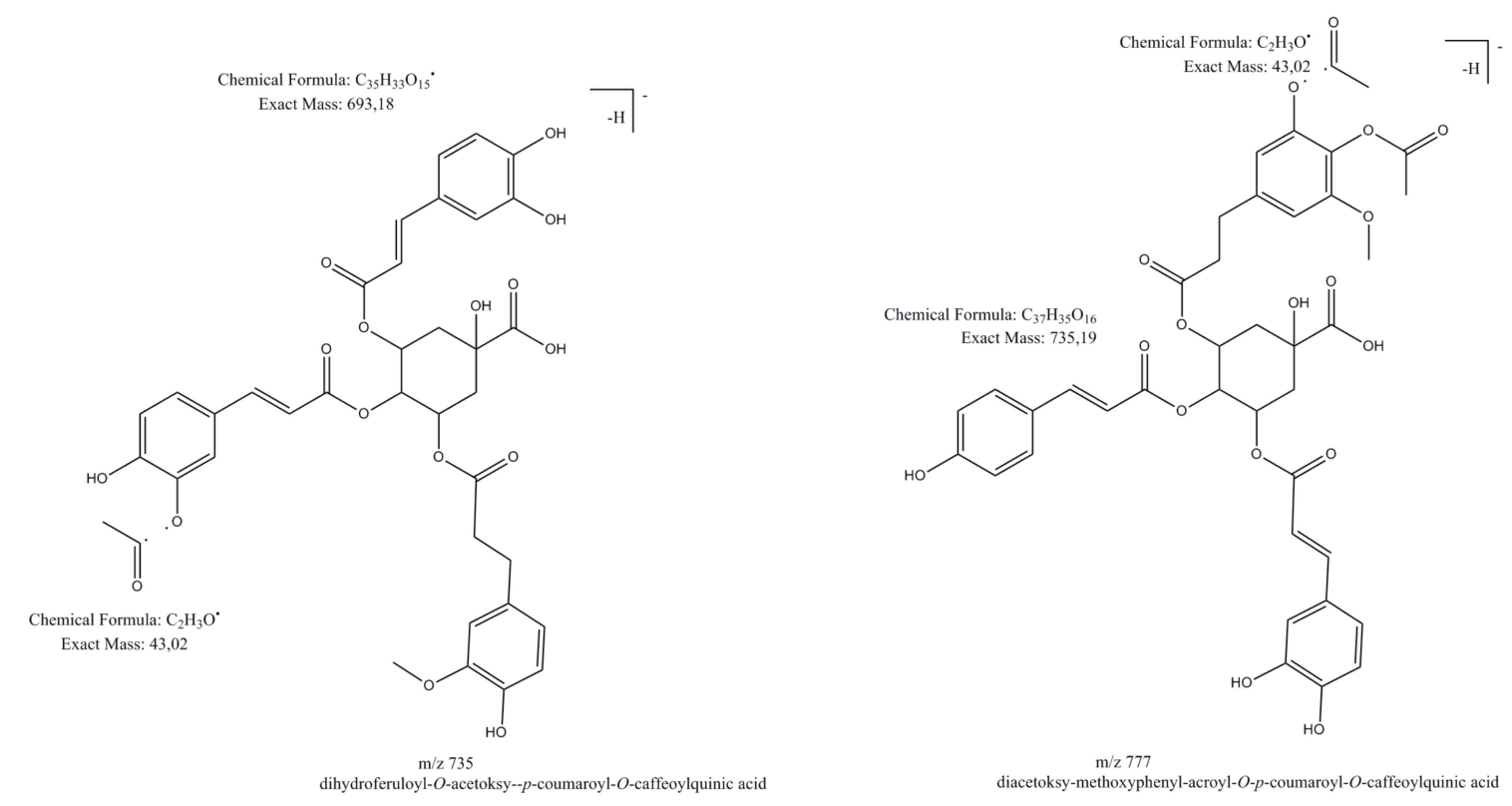

Figure 7. Examples of hydroxycinnamic acids esters.

Deprotonated molecules at $m / z 777[\mathrm{M}-\mathrm{H}]^{-}$(Figure 7) for compounds 65, 70 and 75 showed the most abundant product ion at $m / z 735[\mathrm{M}-\mathrm{H}-42]^{-}$, due to the loss of an acetyl moiety, a product ion at $m / z 693[\mathrm{M}-\mathrm{H}-42 \times 2]^{-}$due to the loss of the next acetyl moiety, product ions at $m / z$ 717 [M - H-42-18 $]^{-}$due to the loss of an acetyl moiety and $\mathrm{H}_{2} \mathrm{O}$. Fragmentation ions at $\mathrm{m} / z 499$ and 337 are characterized as $p$-coumarylquinic acid moieties [37-39]. Based on the fragmentation pattern and comparisons with the literature, compounds 65, 70, 75 were tentatively assigned as (diacetoxy-methoxyphenyl)acroyl-O-p-coumaroyl-O-caffeoylquinic acid and its isomers [18].

Compounds 86, 90, 98, 99, 104 showed deprotonated moleculee at $m / z 819[\mathrm{M}-\mathrm{H}]^{-}$and similar fragmentation ions like compounds $\mathbf{6 5}, \mathbf{7 0}, \mathbf{7 5}$, for example the most abundant product ion at $m / z$

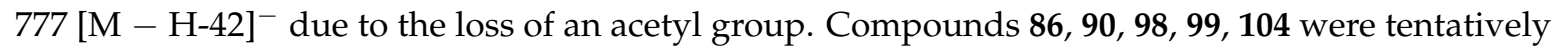
assigned as acetyl derivatives of (diacetoxy-methoxyphenyl)acroyl- $O$ - $p$-coumaroyl- $O$-caffeoylquinic acids [18]. The most abundant peak 118, observed in all dichloromethane fractions and peaks 123, 127 with deprotonated molecules at $m / z 861[\mathrm{M}-\mathrm{H}]^{-}$were described as diacetyl derivatives of (diacetoxy-methoxyphenyl)acroyl-O-p-coumaroyl-O-caffeoylquinic acid. The product ion at $m / z$

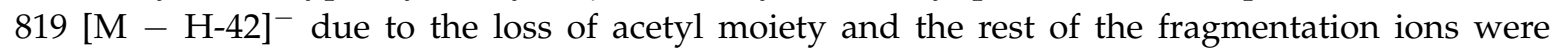
similar to earlier described hydroxycinnamic acid derivatives. Compound 134 observed in the dichloromethane fraction of $R$. sahalinensis with a deprotonated molecule at $m / z 965[\mathrm{M}-\mathrm{H}]^{-}$ and compound $\mathbf{1 3 5}$ observed in the dichloromethane fraction of $R$. x bohemica with a deprotonated molecule at $m / z 995[\mathrm{M}-\mathrm{H}]^{-}$due to the more complex structure was described as derivatives of (diacetoxy-methoxyphenyl)acroyl-O-p-coumaroyl-O-caffeoylquinic acid. However, it can be assumed that compound $\mathbf{1 3 4}$ is a coumaroyl or deohexosyl derivative of compound $\mathbf{8 6}$ or its isomers, due to the loss of the moiety at $m / z 146$ and a product ion at $m / z 819$ [M $-\mathrm{H}-146]^{-}$. The fragmentation of the product ion at $m / z 819$ gave product ions which were similar to those of compound 86 , whereas fragmentation of compound 135 gave the most abundant product ion at $m / z 819$ [M $-\mathrm{H}-176]^{-}$due to the loss of feruloyl or oxyhexosyl moiety. 


\subsubsection{Naphthalene Derivatives}

Compound 56 was characterized by HPLC-DAD-HR-MS analysis in a previous article as torachrysone glucoside [5]. Peak 56 showed deprotonated molecule at $m / z 407$ [M - H] ${ }^{-}$and product ion at $m / z 245[\mathrm{M}-\mathrm{H}-162]^{-}$by cleavage of a glucosidic bond and characteristic for the torachrysone fragmentation ion at $m / z 230[\mathrm{M}-\mathrm{H}-162-15]^{-}$. Torachrysone glucoside was noticed in the acetone extract and dichloromethane fractions of R. japonica and R. x bohemica.

\subsubsection{Other Compounds}

Compound 47 with a deprotonated molecule at $m / z 312[\mathrm{M}-\mathrm{H}]^{-}$was earlier identified as N-trans-feruloyltyramine by HPLC-DAD-HR-MS analysis and described in our previous article [5]. Using a different analytical instrument, based on compound MS, $\mathrm{MS}^{2}$ and $\mathrm{MS}^{3}$ spectra, its identity was confirmed. Moreover, compound 49 exhibited a similar UV/VIS spectrum $\left(\lambda_{\max }\right.$ at 220, 280, $\left.323 \mathrm{~nm}\right)$ and fragmentation pattern to compound $47(\mathrm{~m} / \mathrm{z} 297,178,135)$ and differed from compound 47 by $30 \mathrm{Da}$, which could result from methoxylation. Based on the fragmentation ions and reference [17] compound 49 was tentatively assigned as $N$-feruloylmethoxytyramine, observed in the studied plants for the first time.

Compound 51, because of its deprotonated molecule at $m / z 287$ and product ion at $m / z 269$, the most abundant product ion at $m / z 151$ and product ion at $m / z$ 135, 125, 107 was tentatively identified as cyanidin [18]. Unfortunately due to the fact the UV-vis spectra was recorded in the range of 200-450 nm, it was impossible to get all the maximum spectra of this compound to confirm the assumption. The compound was noticed in fractions of $R . x$ bohemica and $R$. sachalinensis.

Compound 5 observed in fractions of $R$. x bohemica and $R$. sachalinensis with a deprotonated molecule at $m / z 331[\mathrm{M}-\mathrm{H}]^{-}$and the most abundant product ion at $m / z 169[\mathrm{M}-\mathrm{H}-162]^{-}$due to glucosidic bond cleavage was tentatively, based on [11], described as galloyl glucose, earlier observed in $R$. japonica rhizomes.

Compound 159 showed a deprotonated molecule at $m / z 755[\mathrm{M}-\mathrm{H}]^{-}$, product ion at $m / z$ 593 [M - H-162] ${ }^{-}$by cleavage of a glucosidic bond, the most abundant product ion at $\mathrm{m} / z 575$ $[\mathrm{M}-\mathrm{H}-162-18]^{-}$due to the loss of a glucosyl moiety and $\mathrm{H}_{2} \mathrm{O}$, product ion at $m / z 431$ [M $-\mathrm{H}-162$ $\times 2]^{-}$produced by cleavage of two glucosidic bonds. The next fragmentation of the product ion at $m / z 575$ showed that the most abundant fragment ion was $m / z 431$, what together with the rest of the fragmentation ions and characteristic UV/VIS spectrum $\left(\lambda_{\max }\right.$ at $\left.269,333 \mathrm{~nm}\right)$ suggested that peak 159 could be isovitexin or vitexin diglucoside [29,30]. It was observed only in the dichloromethane fraction of R. x bohemica. It was noticed for the first time in this species.

Compound 170 because of its lipophilic character and deprotonated molecule at $m / z 277[\mathrm{M}-\mathrm{H}]^{-}$, product ion at $m / z 259[\mathrm{M}-\mathrm{H}-18]^{-}$due to the loss of $\mathrm{H}_{2} \mathrm{O}$ and the most abundant product ion at $m / z$ $233[\mathrm{M}-\mathrm{H}-44]^{-}$due to loss of $\mathrm{CO}_{2}$ was tentatively assigned as $\alpha$-carboxyethylhydroxychroman [31]. It was observed in the dichloromethane fractions of R. x bohemica and R.sachalinesis.

\subsection{Antioxidant Activities and Polyphenols Content}

Results of bioactivity screening of all 18 extracts and fractions are presented in Table 2.

All studied acetone extracts demonstrated high ability to scavenge the 2,2'-diphenylpicrylhydrazyl radical, comparable to ascorbic acid. Fractionation of extracts allowed us to obtained fractions like the ethyl acetate one with even stronger stable radical scavenging properties.

High ability to scavenge stable radicals was associated with high amount of polyphenols, especially tannins in the studied extract and fractions (Table 3), what was demonstrated by the Spearman Rank Order Correlation in Table 4. 
Table 2. Antioxidant activity of the studied extracts and fractions.

\begin{tabular}{|c|c|c|c|c|c|c|c|c|c|c|c|c|}
\hline \multirow[t]{2}{*}{ Fraction } & \multicolumn{3}{|c|}{$\begin{array}{l}\text { Radical Scavenging Activity DPPH } \\
\left(\mathrm{EC}_{50} \mu \mathrm{g} / \mathrm{mL}\right)\end{array}$} & \multicolumn{3}{|c|}{ Reducing Power AAE (\%) $37^{\circ} \mathrm{C}$} & \multicolumn{3}{|c|}{ Reducing Power AAE (\%) $90^{\circ} \mathrm{C}$} & \multicolumn{3}{|c|}{ LA-Peroxidation $\left(\mathrm{IC}_{50} \mu \mathrm{g} / \mathrm{mL}\right)$} \\
\hline & $R . j$ & R.s & R.b & $R . j$ & R.s & R.b & $R . j$ & R.s & R.b & $R . j$ & R.s & R.b \\
\hline & $9.6 \pm 0.5$ & \pm 0.4 & $6 \pm 0.7$ & \pm 0.3 & $6.0 \pm 0.3$ & $6.4 \pm 0.2$ & $28.5 \pm 1.1$ & $27.9 \pm 1.0$ & $21.4 \pm 1.6$ & $80.3 \pm 2.8$ & $71.6 \pm 2.6$ & $68.9 \pm 1.6$ \\
\hline Dichloron & $202.1 \pm 5.6$ & $56.5 \pm 3.9$ & $63.3 \pm 2.9$ & $2.6 \pm 0.2$ & $1.8 \pm 0.1$ & $1.6 \pm 0.06$ & $11.2 \pm 0.1$ & $12.2 \pm 0.6$ & $10.8 \pm 0.4$ & $401.8 \pm 12.7$ & $112.2 \pm 2.5$ & $153.6 \pm 6.0$ \\
\hline Diethyl ether & $9.3 \pm 0.4$ & $10.2 \pm 0.8$ & $8.8 \pm 0.3$ & $10.2 \pm 0.5$ & $8.3 \pm 0.4$ & $10.9 \pm 0.4$ & $35.0 \pm 1.6$ & $32.6 \pm 1.2$ & $35.4 \pm 1.1$ & $63.8 \pm 2.6$ & $67.3 \pm 1.4$ & $52.1 \pm 2.6$ \\
\hline Ethyl acetate & $6.5 \pm 0.4$ & $4.7 \pm 0.3$ & $6.2 \pm 0.1$ & $13.9 \pm 0.3$ & $16.2 \pm 0.2$ & $16.6 \pm 0.2$ & $38.8 \pm 1.3$ & $44.7 \pm 1.3$ & $36.5 \pm 1.7$ & $45.7 \pm 1.9$ & $32.3 \pm 1.7$ & $40.6 \pm 1.4$ \\
\hline Butanol & $9.1 \pm 0.3$ & $6.9 \pm 0.2$ & $8.1 \pm 0.3$ & $6.6 \pm 0.2$ & $8.2 \pm 0.2$ & $8.1 \pm 0.2$ & $29.0 \pm 1.1$ & $29.4 \pm 0.8$ & $25.7 \pm 1.2$ & $93.2 \pm 3.5$ & $66.2 \pm 2.6$ & $113.4 \pm 4.2$ \\
\hline Water & $58.0 \pm 2.5$ & $35.0 \pm 0.5$ & $57.3 \pm 2.3$ & $0.6 \pm 0.02$ & $1.5 \pm 0.05$ & $0.1 \pm 0.01$ & $13.6 \pm 0.4$ & $16.9 \pm 0.4$ & $12.8 \pm 0.2$ & $650.7 \pm 10.6$ & $635.6 \pm 17.8$ & $690.1 \pm 9.0$ \\
\hline
\end{tabular}

Radical Scavenging activity DPPH for ascorbic acid (as control) EC50 $=8.6 \pm 0.4 \mu \mathrm{g} / \mathrm{mL}$; Reducing power AAE (\%) for quercetin (as control) at $37^{\circ} \mathrm{C}=30.7 \pm 1.2 \mathrm{AAE}(\%)$ and at $90^{\circ} \mathrm{C}=52.0 \pm 2.7 \mathrm{AAE}(\%)$, LA-Peroxidation for quercetin (as control) IC50 = $19.6 \pm 1.1 \mu \mathrm{g} / \mathrm{mL}$. R.j-Reynoutria japonica, R.s-Reynoutria sachalinensis, R.b-Reynoutria $\times$ bohemica. Data were expressed as mean $\pm \mathrm{SD}$, performed in at least three independent experiments, assayed in triplicate. 
Table 3. Total polyphenols and tannins content in studied extracts and fractions. Data were expressed as mean $\pm \mathrm{SD}$, performed in at least three independent experiments, assayed in triplicate.

\begin{tabular}{ccccccc}
\hline \multirow{2}{*}{ Fraction } & \multicolumn{3}{c}{$\begin{array}{c}\text { TPC Total Polyphenols } \\
\text { [GAE] mg/g Fraction }\end{array}$} & \multicolumn{2}{c}{ Tannins Content [GAE] mg/g Fraction } \\
\hline & $R . j$ & $R . s$ & $R . b$ & $R . j$ & $R . s$ & $R . b$ \\
Acetone & $324.1 \pm 9.8$ & $317.7 \pm 14.1$ & $487.7 \pm 11.9$ & $233.3 \pm 6.4$ & $264.0 \pm 7.0$ & $360.0 \pm 6.5$ \\
Dichloromethane & $96.4 \pm 5.6$ & $22.7 \pm 0.9$ & $81.1 \pm 2.7$ & $61.0 \pm 2.9$ & $13.0 \pm 0.4$ & $60.3 \pm 2.7$ \\
Diethyl ether & $469.1 \pm 3.0$ & $355.1 \pm 17.1$ & $615.4 \pm 6.7$ & $338.6 \pm 17.2$ & $241.6 \pm 11.3$ & $509.3 \pm 19.8$ \\
Ethyl acetate & $583.4 \pm 6.5$ & $640.7 \pm 11.0$ & $642.9 \pm 8.9$ & $484.3 \pm 19.1$ & $528.3 \pm 16.9$ & $510.5 \pm 15.8$ \\
Butanol & $307.1 \pm 6.9$ & $352.7 \pm 7.0$ & $286.1 \pm 6.0$ & $258.0 \pm 9.6$ & $315.0 \pm 7.4$ & $243.0 \pm 10.4$ \\
Water & $28.7 \pm 1.5$ & $65.4 \pm 4.5$ & $29.7 \pm 2.2$ & $23.6 \pm 1.1$ & $46.6 \pm 2.0$ & $29.3 \pm 0.6$ \\
\hline
\end{tabular}

Table 4. Spearman Rank Order Correlation. Marked correlations are significant at $p<0.05$.

\begin{tabular}{|c|c|c|c|c|c|c|c|c|}
\hline Variable & $\begin{array}{l}\text { LA-Peroxidation } \\
\text { EC }_{50}\end{array}$ & $\begin{array}{c}\text { DPPH } \\
\text { EC }_{50}\end{array}$ & $\begin{array}{l}\text { Reducing } \\
\text { Power } \\
\text { AAE } 37^{\circ} \mathrm{C}\end{array}$ & $\begin{array}{l}\text { Reducing } \\
\text { Power } \\
\text { AAE } 90^{\circ} \mathrm{C}\end{array}$ & $\begin{array}{c}\text { Total } \\
\text { Polyphenols }\end{array}$ & Tannins & DMACA & HCl-Butanol \\
\hline LA-Peroxidation $\mathrm{EC}_{50}$ & 1000 & 0.751 & -0.904 & -0.874 & -0.823 & -0.804 & -0.938 & -0.300 \\
\hline DPPH EC50 & 0.751 & 1000 & -0.843 & -0.869 & -0.663 & -0.742 & -0.757 & -0.736 \\
\hline Reducing power AAE $37^{\circ} \mathrm{C}$ & -0.904 & -0.843 & 1000 & 0.899 & 0.781 & 0.819 & 0.877 & 0.400 \\
\hline Reducing power AAE $90^{\circ} \mathrm{C}$ & -0.874 & -0.869 & 0.899 & 1000 & 0.795 & 0.810 & 0.917 & 0.411 \\
\hline Total polyphenols & -0.823 & -0.663 & 0.781 & 0.795 & 1000 & 0.939 & 0.779 & 0.259 \\
\hline Tannins & -0.804 & -0.742 & 0.819 & 0.810 & 0.939 & 1000 & 0.738 & 0.378 \\
\hline DMACA & -0.938 & -0.757 & 0.877 & 0.917 & 0.779 & 0.738 & 1000 & 0.272 \\
\hline HCL-Butanol & -0.300 & -0.736 & 0.400 & 0.411 & 0.259 & 0.378 & 0.272 & 1000 \\
\hline
\end{tabular}

These results are in accordance with the above presented phytochemistry of extracts and fractions, where the most antioxidant active ethyl acetate fractions contained numerous polyphenols including procyanidins (Figures 1-3, Table 1). Ethyl acetate fractions, which were the richest in polyphenols and tannins, exhibited also the highest capacity to reduce metal ions (phosphomolybdenum reduction assay) and to prevent the oxidation of linoleic acid. Diethyl ether and butanol fractions of studied species exhibited slightly weaker antioxidant activity, however they also contained significantly lower contents of total polyphenols and tannins (except $R$. x bohemica diethyl ether fraction, where the differences with the ethyl acetate fraction were not significant). Because the results indicated the big impact of tannins on antioxidant activity, what was according with phytochemical analysis, we decided to check the amount of procyanidins in the studied extracts and fractions using the acid butanol method (Bate-Smith method) [40] and DMACA-HCl assay. Results, presented in Figure 8, revealed that ethyl acetate and butanol fractions contained the highest amount of proanthocyanidins, whereas $R$. sachalinensis ethyl acetate and butanol fractions contained significantly higher amount proanthocyanidins than others.

Although the acetone extract of $R$. sachalinensis contained the highest amount of proanthocyanidins, the diethyl ether fraction contained the lowest amounts compared to diethyl ether fractions from other species. This indicates different fractionation efficiency, which could be affected by differences in the composition of the mixtures or different individual structures of the separating compounds. The content of proanthocyanidins in the butanol fractions is very similar to the content in the ethyl acetate fractions of the studied species, despite the fact that the Folin-Ciocalteu assay showed significantly less content of tannins in the butanol fractions than in the ethyl acetate fractions.

It is important to mention that the acid butanol method we used to measure the amount of proanthocyanidins involves depolymerization of the polymer of proanthocyanidins in acid and conversion of the monomers to anthocyanidins, which were spectrophotometrically quantified. Based on our results, we assumed that there are more proanthocyanidins with higher degree of polymerization in the butanol fractions of all studied species than in the ethyl acetate fractions. This assumption agrees with LC-MS analysis in which compounds putatively identified as procyanidin heptamer and octamer were noticed mainly in butanol fractions of studied species. The results from the DMACA assay indicated that in diethyl ether and ethyl acetate fractions are significantly more flavanols than in butanol fractions (Figure 9). 


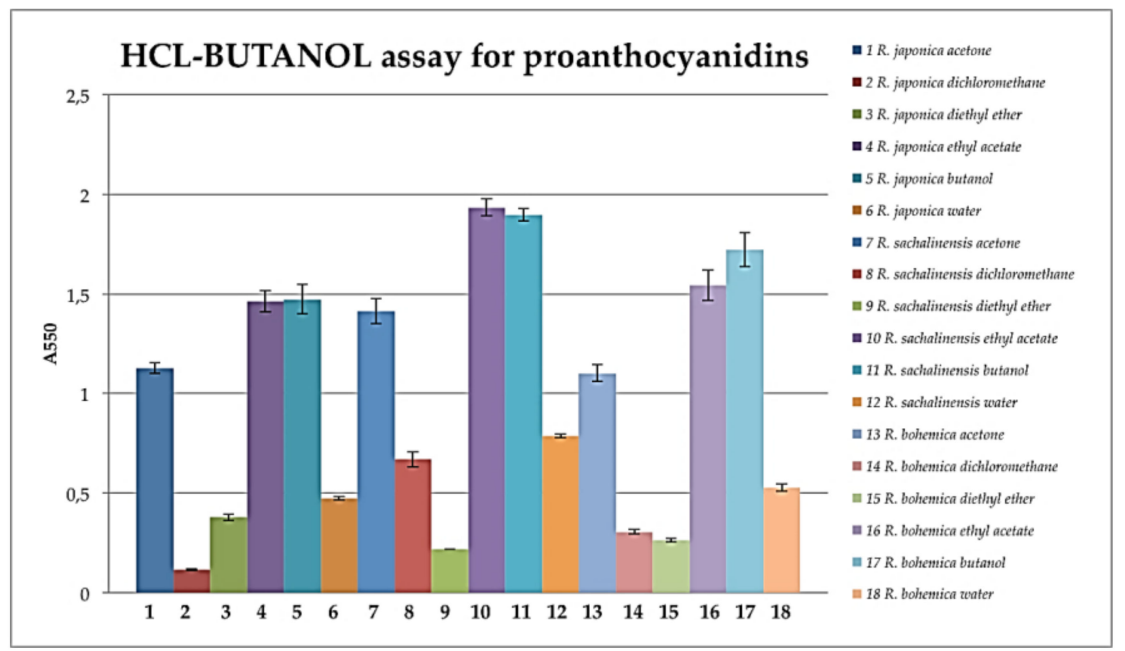

Figure 8. Proanthocyanidins quantified spectrometrically by absorbance at $550 \mathrm{~nm}$ in extracts and fractions. Data were expressed as mean $\pm \mathrm{SD}$, performed in at least three independent experiments, assayed in triplicate.

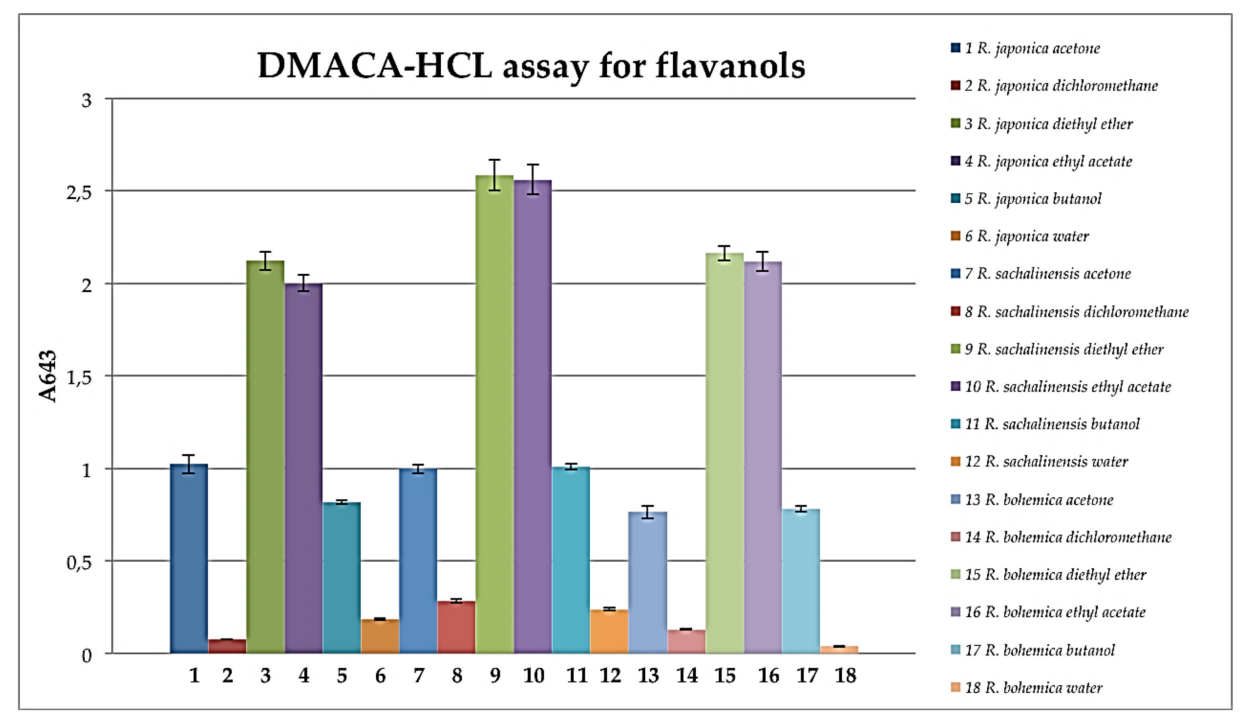

Figure 9. Flavanols quantified spectrophotometrically for absorbance at $643 \mathrm{~nm}$ in extracts and fractions. Data were expressed as mean $\pm \mathrm{SD}$, performed in at least three independent experiments, assayed in triplicate.

4-Dimethylaminocinnamaldehyde (DMACA) reacts with $m$-diphenols to form coloured carbonium ions in acid and this reaction is utilized for the assay of flavanols, because the A-rings of flavanols have $m$-diphenol functionalities [41]. The DMACA reaction affects the C8 position of the A-ring and reacts only with the terminal units of a proanthocyanidins. In this assay, it does not matter how many monomers a proanthocyanidin molecule is made of, but it indicates how many free C8 positions it has. The results agree with the assumption that in the ethyl acetate fractions contain more proanthocyanidin molecules than the butanol ones, but they are made up of fewer monomers. High results in the DMACA assay in diethyl ether fractions may be due to a high content of flavanols other than procyanidins, such as catechin, epicatechin or epicatechin-3-O-gallate what is in accordance with chromatographic analysis of these fractions.

In order to observe relationships between the individual compounds present in the fractions and antioxidant activity, we used chemometric analyses. The principal component analysis (PCA) allowed exploratory analyses of the data which included the results of antioxidant tests and the LC-MS data 
(peak area of compounds), summarizing the multidimensional data in an intelligible way to detect the underlying characteristics and structures of the data (Figure 10).

(A)

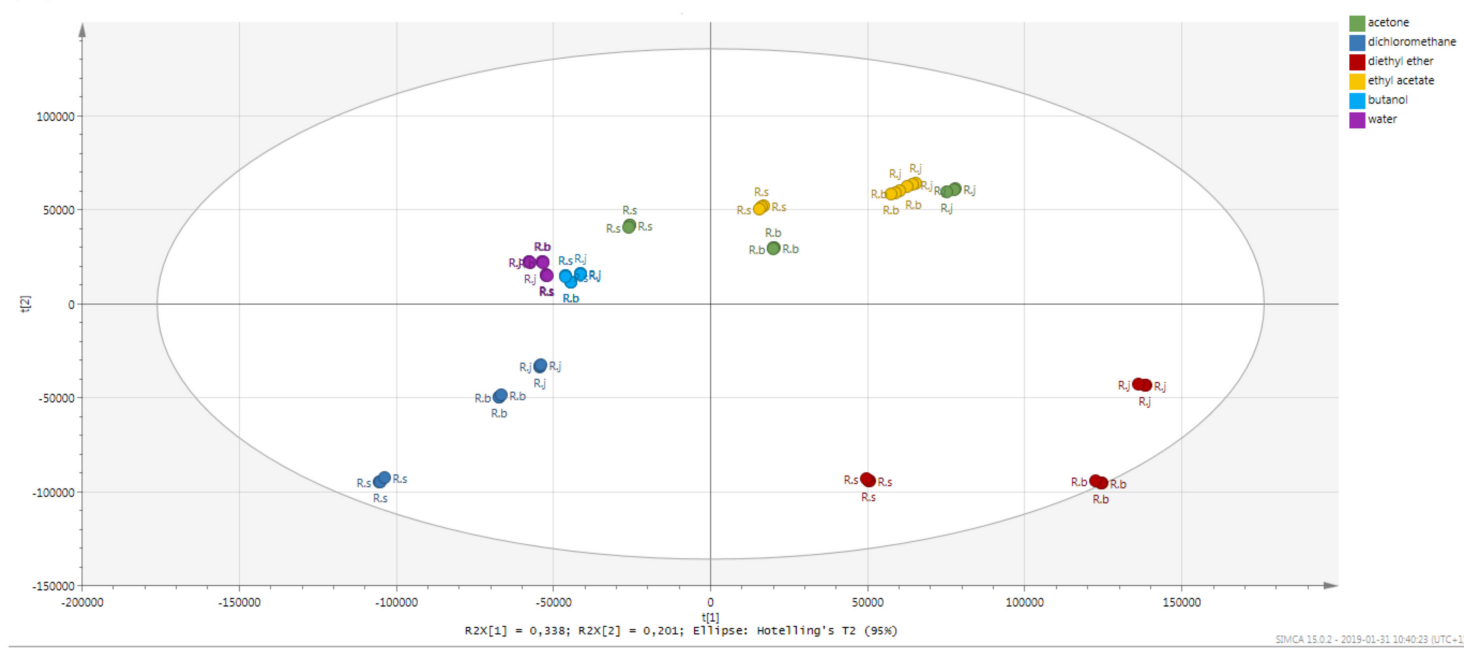

(B)

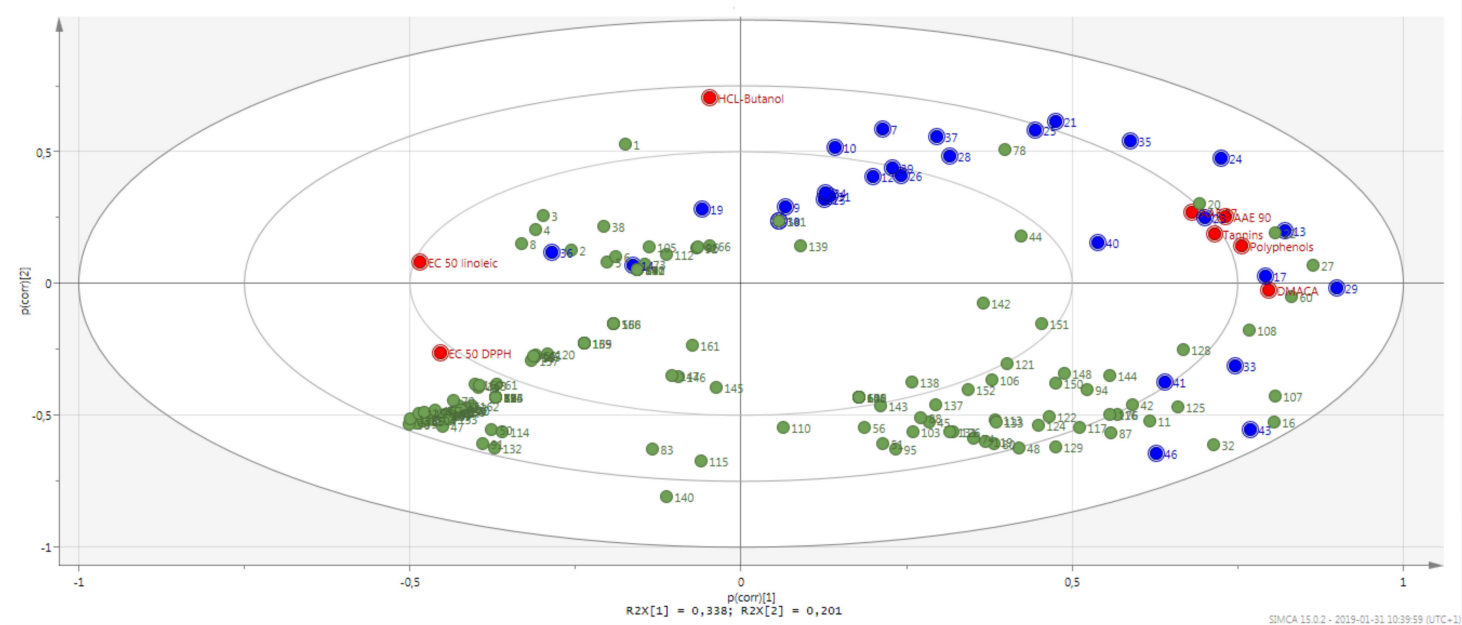

(C)

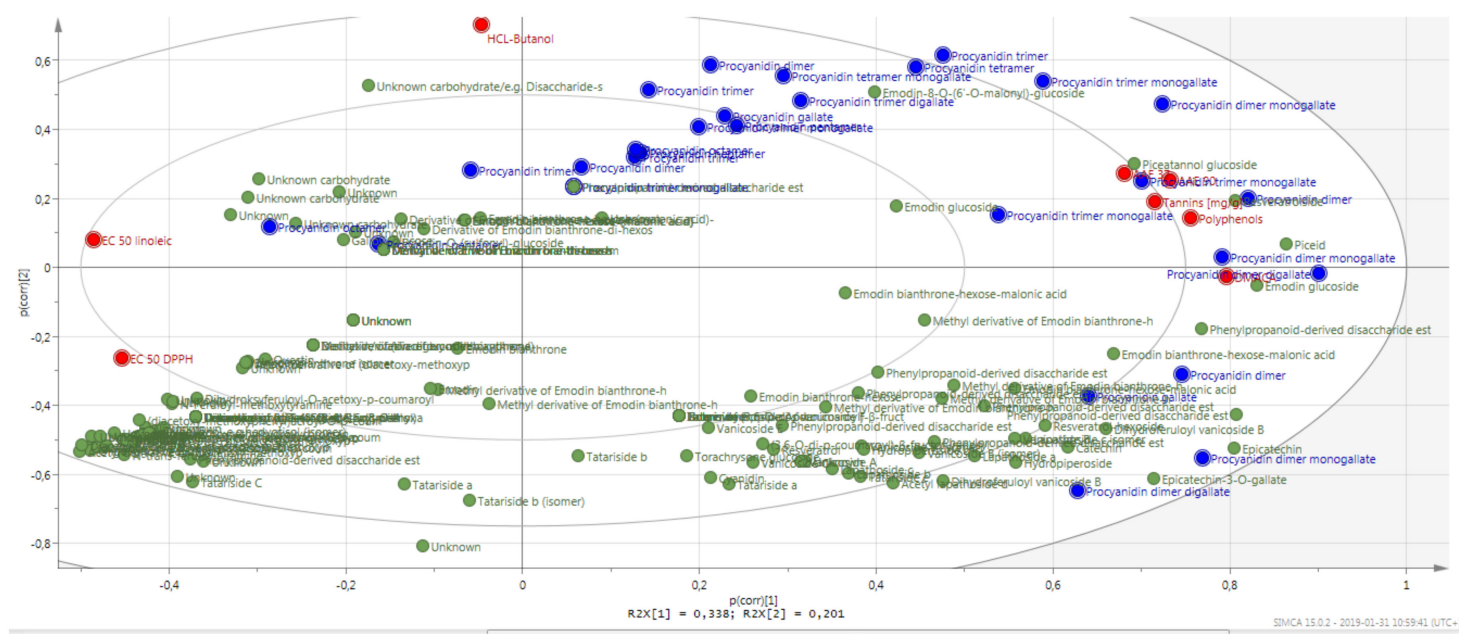

Figure 10. DPPH free radical scavenging activity of vanicoside $\mathrm{A}$, vanicoside $\mathrm{B}, R$. sachalinensis acetone extract and $R$. sachalinensis ethyl acetate fraction with range of concentrations. SC\% percentage of scavenging activity on DPPH radical. The absorbance at $517 \mathrm{~nm}$ was measured after $30 \mathrm{~min}$. 
The visualization of the PCA scores plot shows similarities/dissimilarities between (explained by principal component 1 (PC1)) and within (explained by PC2) the sample clusters. On the PCA score plot all ethyl acetate and diethyl ether fractions with the most antioxidant activity as well as $R . j$ and R.b acetone extracts were located on the right side of the plot. According to the loading plot for this differentiation, compounds located mostly in the right plot are responsible, such as procyanidins (mainly 13- procyanidin dimer, 17- procyanidin dimer monogallate, 29- procyanidin dimer digallate), stilbenes (mainly 20- piceatannol glucoside, 22- resveratrolside, 27- piceid), emodin glucoside (60), as well as almost all performed assays (without $\mathrm{HCl}$-butanol). Dissimilarities between the ethyl acetate and diethyl ether fractions distributed in the third and fourth quadrant are explained by PC2. According to the loading plot the biggest impact on the created ethyl acetate and acetone cluster in the third quadrant had procyanidins and HCl-butanol, whereas for diethyl ether cluster formation phenylpropanoid disaccharide esters were relevant, as well as catechin (11), epicatechin (16), epicatechin-3-O-gallate (32) and some procyanidins (compounds 33, 41, 43, 46). The PCA score plot reveals the difference between $R$. sachalinensis and the more similar to each other $R$. japonica and $R . \mathrm{x}$ bohemica. According to the loading plot, in the case of the acetone extract, ethyl acetate and diethyl ether fractions, dissimilarities are the result of a smaller contribution of PC1, which is in accordance with the phytochemical analysis, where, among others, no stilbenes were observed in the $R$. sachalinensis extract and fractions. Moreover, the loading plot revealed a high correlation of the performed assays (except $\mathrm{HCl}$-butanol) to each other, which agrees with the results in Table 4. Located on the left side of the plot the results from the DPPH assay and linoleic acid peroxidation assay are due to the usage of $\mathrm{EC}_{50}$ as an activity measure (i.e., a lower value of the parameter means a higher activity). Considering the location of AAE 37 and AAE 90 on the loading plot, it can be suggested that there were correlations with procyanidins and some stilbene compounds relatively close located to the AAE 37 and AAE 90 points. In the case of the DPPH assay, a strong correlation is seen mainly with procyanidins, located in the third quadrant of the loading plot, close to the line extension running from the DPPH EC 50 point through point 0 . Similarly in the case of the $\mathrm{EC}_{50}$ values in the linoleic acid peroxidation assay, the correlation seems to be strong also with some of the phenylpropanoid disaccharide esters found in the fourth quadrant of loading plot. These assumptions are consistent with the results presented in Table 5, which shows the strength of the correlations of compounds with antioxidant assays.

The presented statistical analyses show that the high antioxidant activity of fractions and extracts was significantly influenced by procyanidins. Interestingly, stilbenes occurring in a significant amount in the R. japonica and $R$. x bohemica extract and fractions and phenylpropanoid disaccharide esters, especially vanicoside $\mathrm{A}$ and $\mathrm{B}$, occurring in a significant amount in the $R$. sachalinensis extract and fractions turned out to have less influence on antioxidant activity of the studied samples. Considering that the R. sachalinensis ethyl acetate fraction with the most antioxidant activity contained almost only procyanidins and phenylpropanoid disaccharide esters, especially a high amount of vanicoside $A$ and $\mathrm{B}$, we decided to check the DPPH free radical scavenging activity of isolated vanicosides A and B to find out to what extent they affect the fraction activity. Results from the DPPH free radical scavenging activity of vanicoside A, vanicoside B, presented in Figure 11, revealed significantly weaker activity of the tested compounds in relation to the acetone and ethyl acetate $R$. sachalinensis fraction. Thus other compounds must influence the strong fraction activity.

Fan et al. [23] measured the free radical scavenging activity of four phenylpropanoid-derived disaccharide esters obtained from stems of $R$. sachalinensis, which scavenging increased as follows: vanicoside $\mathrm{B}<$ hydropiperoside $<$ lapathoside $\mathrm{C}<$ lapathoside $\mathrm{D}$, whereas $95 \mu \mathrm{g} / \mathrm{mL}$ of vanicoside B demonstrated scavenging about 32\% of DPPH (what was similar to our result) and $95 \mu \mathrm{g} / \mathrm{mL}$ of lapathoside D scavenging about $75 \%$ of DPPH. Taking the above results into account, even the strongest scavenger activity of phenylpropanoid-derived disaccharide esters does not explain the much stronger activity of extracts and fractions of $R$. sachalinensis. 
Table 5. Correlation between the peak area of detected compounds (established by using mass spectral deconvolution) and activity of extracts/fractions (1/EC $50 \mathrm{DPPH}$, Reducing power AAE 37, 90 (\%), $1 / \mathrm{EC}_{50}$ of LA peroxidation) was described with the statistical methods-correlation matrix. In the table are presents only peaks with positive correlation, significant at $p<0.05$.

\begin{tabular}{|c|c|c|c|c|c|}
\hline Nr. & Identification & EC50linoleic & EC50 DPPH & AAE 37 & AAE 90 \\
\hline 9 & Procyanidin dimer & 0.563 & 0.552 & 0.458 & 0.484 \\
\hline 10 & Procyanidin trimer & 0.63 & 0.68 & 0.62 & 0.572 \\
\hline 11 & Catechin & 0.611 & 0.305 & 0.373 & 0.502 \\
\hline 12 & Procyanidin trimer monogallate & 0.635 & 0.646 & 0.665 & 0.601 \\
\hline 13 & Procyanidin dimer & 0.554 & 0.536 & 0.664 & 0.645 \\
\hline 15 & Procyanidin trimer & 0.555 & 0.571 & 0.536 & 0.527 \\
\hline 17 & Procyanidin dimer monogallate & 0.763 & 0.655 & 0.762 & 0.795 \\
\hline 18 & Procyanidin trimer monogallate & 0.494 & 0.504 & 0.446 & 0.445 \\
\hline 20 & Piceatannol glucoside & 0.432 & 0.389 & 0.588 & 0.446 \\
\hline 21 & Procyanidin trimer & 0.48 & 0.512 & 0.6 & 0.501 \\
\hline 22 & Resveratrolside & 0.342 & 0.353 & 0.499 & 0.491 \\
\hline 23 & Procyanidin trimer monogallate & 0.781 & 0.697 & 0.806 & 0.783 \\
\hline 24 & Procyanidin dimer monogallate & 0.687 & 0.684 & 0.758 & 0.734 \\
\hline 25 & Procyanidin tetramer & 0.481 & 0.526 & 0.608 & 0.518 \\
\hline 26 & Procyanidin pentamer & 0.35 & 0.438 & 0.584 & 0.387 \\
\hline 27 & Piceid & 0.34 & 0.319 & 0.48 & 0.466 \\
\hline 28 & Procyanidin trimer digallate & 0.592 & 0.598 & 0.717 & 0.585 \\
\hline 29 & Procyanidin dimer digallate & 0.477 & 0.414 & 0.592 & 0.583 \\
\hline 30 & Procyanidin trimer monogallate & 0.494 & 0.504 & 0.446 & 0.445 \\
\hline 35 & Procyanidin trimer monogallate & 0.746 & 0.719 & 0.764 & 0.721 \\
\hline 37 & Procyanidin tetramer monogallate & 0.682 & 0.701 & 0.729 & 0.643 \\
\hline 39 & Procyanidin gallate & 0.666 & 0.669 & 0.724 & 0.618 \\
\hline 40 & Procyanidin trimer monogallate & 0.716 & 0.561 & 0.753 & 0.636 \\
\hline 78 & Emodin-8-O-(6'-O-malonyl)-glucoside & 0.37 & 0.349 & 0.496 & 0.316 \\
\hline 87 & Hydropiperoside & 0.541 & 0.212 & 0.264 & 0.395 \\
\hline 106 & $\begin{array}{c}\text { Phenylpropanoid-derived } \\
\text { disaccharide esters }\end{array}$ & 0.659 & 0.391 & 0.424 & 0.509 \\
\hline 107 & $\begin{array}{c}\text { Phenylpropanoid-derived } \\
\text { disaccharide esters }\end{array}$ & 0.511 & 0.366 & 0.458 & 0.561 \\
\hline 108 & $\begin{array}{c}\text { Phenylpropanoid-derived } \\
\text { disaccharide esters }\end{array}$ & 0.704 & 0.477 & 0.631 & 0.719 \\
\hline 113 & Vanicoside B (isomer) & 0.501 & 0.166 & 0.198 & 0.338 \\
\hline 116 & Vanicoside B & 0.618 & 0.315 & 0.349 & 0.473 \\
\hline 117 & Lapathoside a & 0.537 & 0.209 & 0.263 & 0.394 \\
\hline 121 & $\begin{array}{c}\text { Phenylpropanoid-derived } \\
\text { disaccharide esters }\end{array}$ & 0.579 & 0.41 & 0.407 & 0.511 \\
\hline 122 & $\begin{array}{l}\text { Phenylpropanoid-derived } \\
\text { disaccharide esters }\end{array}$ & 0.556 & 0.289 & 0.358 & 0.447 \\
\hline 124 & Vanicoside B (isomer) & 0.564 & 0.217 & 0.284 & 0.403 \\
\hline 125 & Dihydroferuloylvanicoside B & 0.624 & 0.341 & 0.39 & 0.54 \\
\hline 141 & $\begin{array}{l}\text { Phenylpropanoid-derived } \\
\text { disaccharide esters }\end{array}$ & 0.494 & 0.504 & 0.446 & 0.445 \\
\hline
\end{tabular}

Meanwhile, according to the literature, the strong antioxidant activity of $R$. japonica rhizomes is often associated with high amounts of stilbenes, mainly resveratrol [6,42,43]. However, there is some evidence that other compounds are co-responsible for high antioxidant activity of rhizomes of Reynoutria japonica. As shown by Pan et al. [9], ethanol extract from Polygon cuspidati rhizoma was stronger than resveratrol in DPPH and hydroxyl radical scavenging, metal reducing capacity, and preventing of polyunsaturated lipids peroxidation. Also, in the study of Lee et al. [7], no correlation was observed between the content of resveratrol or emodin and antioxidant activity. These results suggest the importance other polyphenols or another group of compounds for determination of antioxidant properties of $R$. japonica rhizomes. Research of Lachowicz et al. [44] indicates a significant 
influence of procyanidins on antioxidant activity; flavan-3-ols derivatives such as catechins and procyanidins as well trans-piceid and trans-resveratrolside had greater radical scavenging capacity than other compounds observed in $R$. japonica and $R$. sachalinensis extracts.

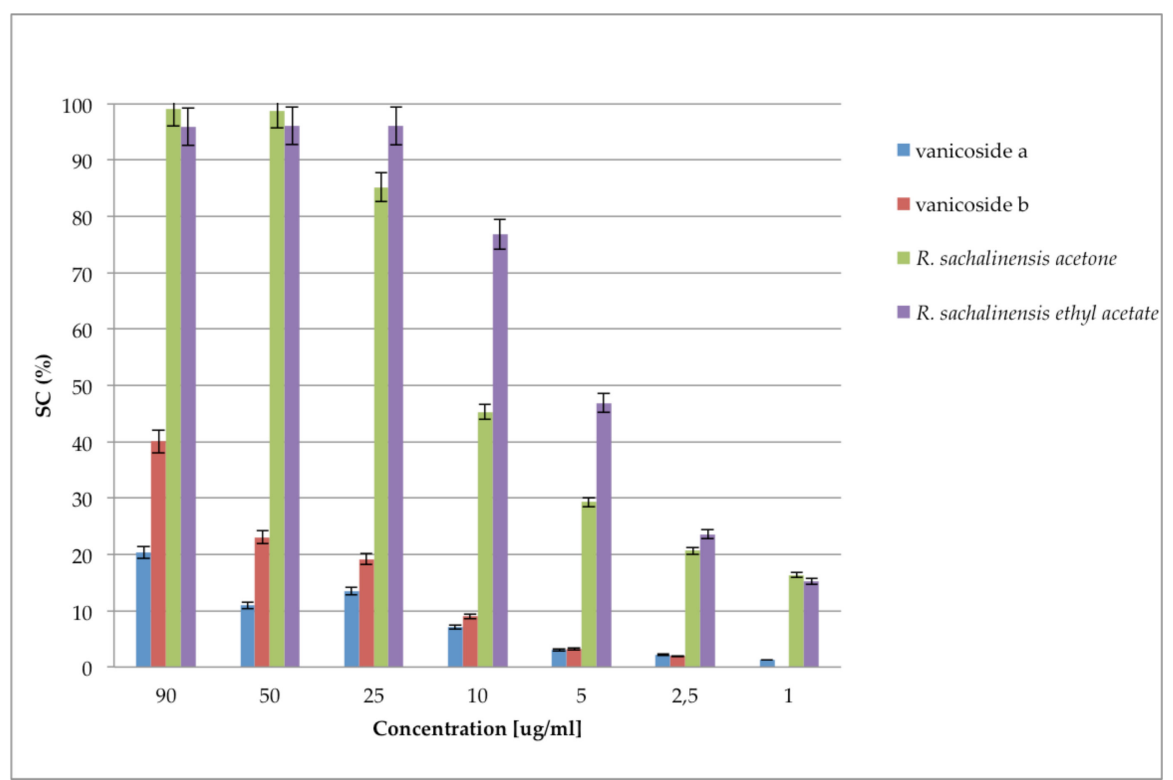

Figure 11. Principal components analysis (PCA) plots indicating the general grouping of the variables in the data sets of extracts (green-acetone) and fractions (blue-dichloromethane, red-diethyl ether, yellow-ethyl acetate, blue-butanol, purple-water) from R.j-Reynoutria japonica, R.b-Reynoutria x bohemica, R.s-Reynoutria sachalinensis in three independent experiments. (A) The PCA score plot of the LC-MS data and antioxidant assay illustrates the general clustering of the variables. The scores plot was computed using the first two principal components (PC1 vs. PC2). The circle in the score plot represents Hoteling's T2 with 95\% confidence interval. R2X(cum) $=0.911$, Q2 (cum) $=0.693$ for 7 components. (B) Loading plot of PCA results obtained from LC-MS data and antioxidant assay. Numbers represent the compounds listed in Table 1. Blue points represents procyanidins, red-antioxidant tests, green-all compounds without procyanidins. (C) Enlarged image of the loading plot of PCA with named compounds.

DPPH scavenging activity and inhibition of lipid peroxidation of proanthocyanidins was investigated in numerous studies $[45,46]$. Proanthocyanidins are strong DPPH scavengers, e.g., the DPPH $\mathrm{IC}_{50}$ values for procyanidin A2 and procyanidin B2 are 2.29 and $3.14 \mu \mathrm{g} / \mathrm{mL}$, respectively [47]. The scavenging activity of proanthocyanidins increases with the number of hydroxyl groups, especially if they are in the benzene ortho position.

Furthermore, polymerization up to trimers increases, but further polymerization decreases the scavenging activity. Higher scavenging activity was found for galloylated procyanidins [45,46]. Among various type of polyphenols, dimeric procyanidins were the most active in scavenging of ABTS and hypochlorous acid and in the FRAP test, followed by flavanols, hydroxycinnamic acids, simple phenolic acids [48]. Taking the above into account it is very likely that procyanidins, including many procyanidins gallate derivatives in the ethyl acetate fractions from the studied Reynoutria rhizomes were largely responsible for strong radical scavenging activity. Proanthocyanidins are also good inhibitors of lipid peroxidation, with potency similar or higher than Trolox and vitamin E [45].

Total antioxidant capacity expressed as the ascorbic acid equivalent (AAE) was based on the reduction of $\mathrm{Mo}(\mathrm{VI})$ to $\mathrm{Mo}(\mathrm{V})$ at acidic $\mathrm{pH}$ by the extracts and fractions and the formation of a green phosphate/ $\mathrm{Mo}(\mathrm{V})$ complex [49]. It appears that unlike ascorbic acid, the compounds (including proanthocyanidins) in the fractions reduced the Mo ions only at a higher $\left(90^{\circ} \mathrm{C}\right)$ temperature (Table 2$)$. The result may be due to the degradation of high polymerized procyanidins at high temperatures and the formation of less polymerized, more active dimer procyanidins. This assumption confirms the 
study of Luo et al. [50] which developed method for degradation of grape proanthocyanidin polymers into oligomers by sulphurous acid in high temperature $\left(60-80^{\circ} \mathrm{C}\right)$ which resulted in many individual procyanidins dimers and trimers. It was also observed that high polymeric procyanidins exhibited lower values of their half-life times in higher temperature than dimeric procyanidins [51].

Rhizomes of $R$. japonica are known as good source of stilbenes [52-55] and antraquinones [53-56]. The European [3] and Chinese Pharmacopeias [57] require determining the content of two compounds, emodin and piceid, in rhizomes of $R$. japonica. However, results of our study suggest that procyanidins should also be considered as compounds affecting the total antioxidant potential of the raw material.

\section{Materials and Methods}

\subsection{Plant Material}

We collected the plant raw material (rhizomes) from a wild population in the disturbed urban areas in the city of Wroclaw (Poland). The rhizomes were harvested in September 2016, when the plants were just before the onset of dormancy. Aerial parts growth and development was completed, leaves still green and fruit abscission beginning. The precise locations of the collection sites are as follows:

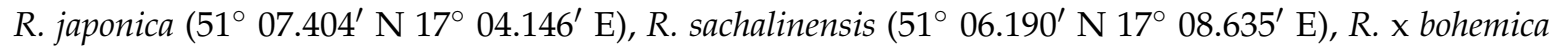
$\left(51^{\circ} 05.666^{\prime} \mathrm{N} 17^{\circ} 01.746^{\prime} \mathrm{E}\right.$ ). Identity of the species was confirmed by Botanical Garden of Medicinal Plants Herbarium staff (Klemens Jakubowski, MSc Botany) based on morphology of the vegetative and generative organs (according to available floras). Voucher specimens were deposited in the Botanical Garden herbarium under deposition numbers AAB1022, AAB1023, AAB1024. The extraction and further sample processing were performed as described previously [5]. In brief, $400 \mathrm{~g}$ of air-dried and powdered rhizomes of all three species were extracted four times ( $2 \mathrm{~h}$ each, drug-to-solvent ratio 1:5) with $70 \%$ aqueous acetone using an ultrasonic bath (Intersonic IS-36, Olsztyn, Poland). The solvent was evaporated under reduced pressure in a rotary evaporator and $73.75 \mathrm{~g}, 70.38 \mathrm{~g}, 79.87 \mathrm{~g}$ of $R$. japonica, $R$. sachalinensis and $R . \mathrm{x}$ bohemica acetone dried extracts were obtained, respectively. Fifty $\mathrm{g}$ of the raw $70 \%$ acetone extract were suspended in water $(500 \mathrm{~mL})$ and partitioned between dichloromethane $\left(\mathrm{CH}_{2} \mathrm{Cl}_{2}\right)$, diethyl ether $\left(\mathrm{Et}_{2} \mathrm{O}\right)$, ethyl acetate $(\mathrm{AcOEt})$ and finally butanol $(n-\mathrm{BuOH})$ affording $0.97,1.05$, $5.11,18.91 \mathrm{~g}$ of each dried fraction for $R$. sachalinensis, 2.29, 3.09, 6.80, $13.54 \mathrm{~g}$ for $R$. japonica and 1.42, $1.835,8.68,14.3 \mathrm{~g}$ for $R . \mathrm{x}$ bohemica and for the all-water residue fraction. Obtained dried extracts and fractions were weighed accurately, dissolved in $80 \% \mathrm{MeOH}$ in volumetric flasks to get a $5 \mathrm{mg} / \mathrm{mL}$ concentration. Before injection into the HPLC system (Dionex, Idstein, Germany), the solutions were filtered through a $0.22 \mu \mathrm{m}$ Chromafil syringe polyester membrane (Macherey-Nagel, Düren, Germany) directly to autosampler vials and stored in darkness at $4{ }^{\circ} \mathrm{C}$. The minimum number of replicates for HPLC analyses was three.

\subsection{Reagents}

2,2-Diphenyl-1-picrylhydrazyl (DPPH), thiobarbituric acid (TBA), and hide powder were purchased from Sigma-Aldrich (Steinheim, Germany). Linoleic and gallic acid were purchased from Fluka AG (St. Gallen, Switzerland), and trichloroacetic acid from Ubichem (Redditch, UK). Vanicoside $A$ and vanicoside $B$ were earlier isolated according procedure described in previous article [5]. All other reagents and solvents were obtained from Avantor-POCh, (Gliwice, Poland).

\subsection{DPPH Scavenging Assay}

The ability to scavenge the DPPH free radical was monitored according to a modified method of [58]. Briefly, DPPH solution $(0.3 \mathrm{mM})$ was prepared in methanol. The extract and fractions were dissolved in a mixture of methanol and water $(9: 1, v / v)$ to obtain stock solution $(1 \mathrm{mg} / \mathrm{mL})$. Then each stock solution was diluted to obtain final concentrations of $1-250 \mu \mathrm{g} / \mathrm{mL}$ in the assay mixture. DPPH solution $(125 \mu \mathrm{L})$ and $125 \mu \mathrm{L}$ of the test extract and fractions at different concentrations were added to a 96- well plate. The absorbance at $517 \mathrm{~nm}$ was measured $30 \mathrm{~min}$ after mixing using a 
microplate reader ( $\mu$ QUANT, BioTek, Winooski, VT, USA). The percentage of scavenged DPPH was then calculated according to Equation (1):

$$
\% \mathrm{DPPH}=((\mathrm{Abt}-\mathrm{Abr}) / \mathrm{Ab} 0) \times 100
$$

where Abt is the absorbance of DPPH solution with the test extracts, Ab0 is the absorbance of DPPH solution with a mixture of methanol and water $(9: 1, v / v)$ and Abr is the absorbance of the test extract solution with the addition of methanol. The antiradical activity of extracts was expressed as an $\mathrm{EC}_{50}$ value.

\subsection{Phosphomolybdenum Reduction Assay}

The antioxidant capacity of the extract and fractions was assessed as described by Prieto et al. [49], with modifications. Extract and fractions were dissolved in a mixture of methanol and water $(9: 1 \mathrm{v} / v)$ to obtain stock solution $(5 \mathrm{mg} / \mathrm{mL})$. Then each stock solution was diluted to obtain final concentrations of $10-500 \mu \mathrm{g} / \mathrm{mL}$ in the assay mixture. The extract and fractions were combined with the reagent solution containing ammonium molybdate $(4 \mathrm{mM})$, sodium phosphate $(28 \mathrm{mM})$ and sulfuric acid $(600 \mathrm{mM})$. The reaction mixture was incubated in a water bath at either $37^{\circ} \mathrm{C}$ or $90^{\circ} \mathrm{C}$ for $90 \mathrm{~min}$. The absorbance of the colored complex was measured at $695 \mathrm{~nm}$. The antioxidant activity was compared with that of ascorbic acid in the same concentration range and was expressed as the ascorbic acid equivalents (AAE).

\subsection{Inhibition of Linoleic Acid Peroxidation}

The procedure of Wozniak et al. [59] using Fenton reaction- induced lipid peroxidation, has been adapted for this assay. The extract and fractions dissolved in water, achieved a concentration range of $10-500 \mu \mathrm{g} / \mathrm{mL}$ in the assay mixture. Each fraction $(150 \mu \mathrm{L})$ was mixed with $500 \mu \mathrm{L}$ phosphate buffer (0.1 M, pH 7.4), and $550 \mu \mathrm{L}$ linoleic acid emulsion (linoleic acid mixed with Tween 80, 3:1, w/w); next $1.12 \mathrm{~g}$ emulsion was mixed with $50 \mathrm{~mL} 0.1 \mathrm{M}$ phosphate buffer (pH 7.4)), and $150 \mu \mathrm{L} 10 \mathrm{mM}$ ascorbic acid. The peroxidation was started with the addition of $150 \mu \mathrm{L} 10 \mathrm{mM} \mathrm{FeSO}_{4}$. The reaction mixture was incubated for $90 \mathrm{~min}$. at $37^{\circ} \mathrm{C}$. Thereafter, $1.5 \mathrm{~mL}$ of $10 \%$ ice cold trichloroacetic acid was added and $1.5 \mathrm{~mL}$ of $1 \%$ thiobarbituric acid in $50 \mathrm{mM} \mathrm{NaOH}$. The samples were heated in a water bath at $90{ }^{\circ} \mathrm{C}$ for $10 \mathrm{~min}$. After cooling the samples, $2 \mathrm{~mL}$ of $n$-BuOH was added and mixed well. The absorbance was read at $532 \mathrm{~nm}$ after transferring $300 \mu \mathrm{L}$ of $\mathrm{BuOH}$ phase from samples to the 96-well plate. The percentage of linoleic acid peroxidation inhibition was calculated as in [59] using appropriate controls. The inhibition of linoleic acid peroxidation of extracts was expressed as an $\mathrm{IC}_{50}$ value.

\subsection{Total Polyphenols and Tannins Content}

Total phenolic content was determined with the Folin-Ciocalteu reagent according to a procedure described previously [60]. Tannin compounds were measured by parallel experiments with extracts vortexed for $1 \mathrm{~h}$ with $10 \mathrm{mg} / \mathrm{mL}$ hide powder. The results were expressed as gallic acid equivalents according to the standard gallic acid calibration curve. Total tannins were calculated by subtraction of polyphenols non-absorbed by hide powder from the total phenol content.

\subsection{HCl-Butanol Assay}

Quantification of proanthocyanidins (i.e., procyanidins and delphinidins) in the Reynoutria species extracts and fractions was performed in three replicates using the acid butanol method (Bate-Smith method) [40]. (Proanthocyanidins contained in $1 \mathrm{~mL}$ of Reynoutria species extracts or fractions (at $1 \mathrm{mg} / \mathrm{mL}$ ) were oxidatively cleaved to anthocyanidins (i.e., cyanidins and delphinidins) at $95{ }^{\circ} \mathrm{C}$ for $50 \mathrm{~min}$ by adding $6 \mathrm{ml}$ of acid- butanol reagent (butanol/12 $\mathrm{N} \mathrm{HCl} ; 95 / 5 ; v / v)$ and $200 \mu \mathrm{L}$ of $2 \%(w / v)$ $\mathrm{NH}_{4} \mathrm{Fe}^{\mathrm{III}}\left(\mathrm{SO}_{4}\right)_{2} \times 12 \mathrm{H}_{2} \mathrm{O}$ (in $2 \mathrm{~mol} / \mathrm{L} \mathrm{HCl}$ ). The reaction mixture was cooled and anthocyanidins 
quantified spectrometrically for absorbance at $550 \mathrm{~nm}$. Blank spectra were obtained for each extract before boiling.

\subsection{DMACA-HCl Assay for Flavanols}

Reynoutria species extracts or fractions $(770 \mu \mathrm{L}$, at $0.1 \mathrm{mg} / \mathrm{mL})$ were mixed with methanol $(385 \mu \mathrm{L})$ and DMACA reagent $(192 \mu \mathrm{L})$, left at room temperature for $20 \mathrm{~min}$ and the absorbance at $643 \mathrm{~nm}$ was measured [61]. The DMACA reagent was prepared immediately before use, containing $2 \%(w / v)$ DMACA in a cold mixture of methanol and $6 \mathrm{M} \mathrm{HCl}(1: 1, v / v)$.

\subsection{HPLC-MS Apparatus}

For the HPLC analyses, we used Ultimate 3000 series system (Dionex, Idstein, Germany) consisting of dual low-pressure gradient pump with vacuum degasser, an autosampler, a thermostatic column compartment, a diode array detector, an Amazon SL ion trap mass spectrometer with the ESI ion source (Bruker Daltonik, Bremen, Germany) and Corona Ultra RS charged aerosol detector (Thermo Scientific, Bellefonte, PA, USA).

\subsection{HPLC-DAD-MS Conditions}

For separation, we used the Kinetex XB C18 $150 \mathrm{~mm} \times 2.1 \mathrm{~mm} \times 1.7 \mu \mathrm{m}$ analytical column (Phenomenex, Torrance, CA, USA), maintained at $25^{\circ} \mathrm{C}$. The following multi-step gradient was used: 0-50 $\min 15-70 \%$ B, 50-55 $\min 70-95 \%$ B, 55-60 $\min 95 \%$ B. The mobile phase " $A$ " $(0.1 \% \mathrm{HCOOH}$ in water), mobile phase " $\mathrm{B}$ " $(0.1 \% \mathrm{HCOOH}$ in $\mathrm{MeCN})$, the flow rate was $0.3 \mathrm{~mL} / \mathrm{min}$ during analysis. $4 \mu \mathrm{L}$ of each sample was injected to the column by the autosampler. The column was equilibrated for 10 min between injections. UV-vis spectra (Dionex, Idstein, Gemany) were recorded in the range of 200-450 nm. The eluate was introduced into mass spectrometer (Bruker Daltonik, Bremen, Germany) in splitless mode. The parameters for ESI source were: nebulizer pressure $40 \mathrm{psi}$; dry gas flow $9 \mathrm{~L} / \mathrm{min}$; dry temperature $300^{\circ} \mathrm{C}$; and capillary voltage $4.5 \mathrm{kV}$. Analysis was carried out using scan from $\mathrm{m} / \mathrm{z}$ 70 to 2200. Compounds were analyzed in negative ion mode. The parameters for Dissect:Internal $\mathrm{S} / \mathrm{N}$ threshold -5; Max. number of overlapping compounds-3; Spectrum type-auto; Cut-off intensity $-0.1 \%$. The identification of constituents found in plant materials was based on DAD and negative ionization mode MS spectra.

\subsection{Statistical Analysis}

Each of the antioxidant tests and analysis of total polyphenols and tannins was made in three independent experiments, assayed in triplicate. Significant differences $(p \leq 0.05)$ between mean values were evaluated by one-way ANOVA and Duncan's multiple range test using Statistica 13.1 (Statsoft, Krakow, Poland); results are given in Supplementary Materials. Spearman's rank order correlation were calculated using Statistica 13.1 Correlation between the peak area of detected compounds (established by using mass spectral deconvolution) and activity of extracts/fractions (1/EC 50 DPPH, Reducing power AAE 37, 90 (\%), 1/EC50 of LA peroxidation) was described with the statistical methods-correlation matrix using Statistica 13.1. Mass spectral deconvolution-the dissect command in Data Analysis TM software (version 4.2, Bruker Daltonics, Billerica, MA, USA) was used to automatically find peak area of compounds on an LC-MS chromatogram trace. The Dissect algorithm utilises fuzzy logic algorithms, which allow a peak separation process to be run without the need for user interaction or any prior information. The parameters for Dissect algorithm: Internal $\mathrm{S} / \mathrm{N}$ threshold -5; Max. number of overlapping compounds-3; Spectrum type-auto; Cut-off intensity $-0.1 \%$. The Principal Component Analysis (PCA) by involved of the LC-MS data (peak area of detected compounds established by using mass spectral deconvolution) and antioxidant assays was performed using Simca-P software (version 15.0.2, Umetrics, Umea, Sweden). Pareto (Par) scaling method with centered and normalized in units of standard deviation were applied to PCA. 


\section{Conclusions}

Fractionation of Reynoutria species extracts allowed us to evaluate compounds present in studied raw materials even in small amounts. HPLC/UV /ESI-MS analysis revealed 171 compounds, a total number of 134 constituents were annotated unambiguously (20) or tentatively (114).

Many of identified compounds were observed for the first time in the studied materials. The rhizomes of all species are a rich source of proanthocyanidins. We confirmed the presence of procyanidins with high degree of polymerization, up to decamers, in the rhizomes of $R$. japonica and brought new data on the presence of these compounds in other Reynoutria species. A procyanidin trimer digallate was described for the first time in the studied plants. Moreover, we suggest a presence of new, for these species, dianthrone glycosides (emodin bianthrone, emodin bianthrone-hexose, emodin bianthrone-dihexose, emodin bianthrone-hexose-malonic acid, emodin bianthrone-hexose-(malonic acid)-hexose and their methyl or undefined derivatives) that, however, need to be confirmed by isolation and structure elucidation. Fractionation has also allowed to observe the numerous and previously unrecorded phenylpropanoid disaccharide esters (tatariside E, tatariside A, tatariside C, lapathoside B, hydropiperoside B, vanicoside $\mathrm{E}$ their isomers and undefined derivatives of phenylpropanoid disaccharide esters) and hydroxycinnamic acid derivatives (dihydroksyferuloyl-O-acetoxy- $p$-coumaroyl-O-caffeoylquinic acid, (diacetoxymethoxyphenyl) acroyl-O-p-coumaroyl-O-caffeoylquinic acid and its acetyl derivatives), mainly in $R$. sachalinensis. Furthermore, compounds tentatively annotated as lignin oligomers (trimer lignin $\beta-O-4-$ linked $S$ unit with syringaresinol $\left[S-\left(\beta-O-4^{\prime}\right)-S-\left(\beta-\beta^{\prime}\right)-S\right]$, tetramer lignin, $S-\left(8-O-4^{\prime}\right)-S-\left(8-O-4^{\prime}\right)-S-\left(8-8^{\prime}\right)-S$, derivative of lignin-S(8-8)S, hedyotisol and its isomers) were observed for the first time in the dichloromethane fractions obtained from the studied species. Other compounds that have been observed for the first time are: $N$-feruloylmethoxytyramine, isovitexin or vitexin diglucoside and slightly suggested: $\alpha$-carboxyethylhydroxychroman and cyanidin.

The rhizomes of all Reynoutria species exhibited strong antioxidant activity. The ethyl acetate fractions, rich in proanthocyanidins, also in galloylated form, were the most active in all antioxidant tests. Statistical analysis demonstrated that proanthocyanidins should be taken considered important contributors to the total antioxidant capacity.

Supplementary Materials: The following are available online at http:/ /www.mdpi.com/1420-3049/24/6/1136/ s1, Table S1: Multi-component one-way ANOVA results for Table 2. Table S2: Multi-component one-way ANOVA results for Table 3, Figures 8 and 9.

Author Contributions: Conceptualization, I.N.-H. and A.M.; Data curation, I.N.-H.; Formal analysis, I.N.-H., S.Ś. and J.H.; Funding acquisition, I.N.-H. and A.M.; Investigation, I.N.-H. and A.M.; Methodology, I.N.-H., S.G. and A.M.; Project administration, A.M.; Resources, S.G. and A.M.; Software, S.Ś.; Supervision, S.S. and A.M.; Validation, I.N.-H., S.Ś., S.G. and A.M.; Visualization, I.N.-H., S.G. and J.H.; Writing-original draft, I.N.-H.; Writing-review \& editing, I.N.-H., J.H. and A.M.

Funding: The study is supported by the Polish National Research Center (NCN) 'Preludium' grant no. 2012/07/N/NZ7/02420 as well as WMU grant \#ST.D.030.17.028.01. The APC was funded by Wroclaw Medical University.

Acknowledgments: I.N.H. and A.M. received support from Wroclaw Medical University for the research stay at the department of Pharmacognosy, Warsaw Medical University. Support to Botanical Garden of Medicinal Plants Herbarium by Polish Ministry of Science and Higher Education grant No. 215259/E-394/SPUB/2016/1 is acknowledged.

Conflicts of Interest: The authors declare no conflict of interest. The funders had no role in the design of the study; in the collection, analyses, or interpretation of data; in the writing of the manuscript, or in the decision to publish the results. 


\section{References}

1. Peng, W.; Qin, R.; Li, X.; Zhou, H. Botany, phytochemistry, pharmacology, and potential application of Polygonum cuspidatum Sieb.et Zucc.: A review. J. Ethnopharmacol. 2013, 148, 729-745. [CrossRef] [PubMed]

2. Truong, V.L.; Jun, M.; Jeong, W.S. Role of resveratrol in regulation of cellular defense systems against oxidative stress. BioFactors 2018, 44, 36-49. [CrossRef] [PubMed]

3. European Pharmacopoeia, 9th ed.; Council of Europe: Strasbourg, France, 2017; pp. 1481-1483.

4. Eom, M.R.; Weon, J.B.; Jung, Y.S.; Ryu, G.H.; Yang, W.S.; Ma, C.J. Neuroprotective compounds from Reynoutria sachalinensis. Arch. Pharm. Res. 2017, 40, 704-712. [CrossRef] [PubMed]

5. Nawrot-Hadzik, I.; Granica, S.; Domaradzki, K.; Pecio, Ł.; Matkowski, A. Isolation and Determination of Phenolic Glycosides and Anthraquinones from Rhizomes of Various Reynoutria Species. Planta Med. 2018, 84, 1118-1126. [CrossRef] [PubMed]

6. Matkowski, A.; Jamiołkowska-Kozlowska, W.; Nawrot, I. Chinese medicinal herbs as source of antioxidant compounds-Where tradition meets the future. Curr. Med. Chem. 2013, 20, 984-1004. [PubMed]

7. Lee, M.H.; Kao, L.; Lin, C.C. Comparison of the antioxidant and transmembrane permeative activities of the different Polygonum cuspidatum extracts in phospholipid-based microemulsions. J. Agric. Food Chem. 2011, 59, 9135-9141. [CrossRef] [PubMed]

8. Ding, X.P.; Zhang, C.L.; Qi, J.; Sun, L.Q.; Qin, M.J.; Yu, B.Y. The Spectrum-Effect integrated fingerprint of Polygonum cuspidatum based on HPLC-diode array detection-flow injection-chemiluminescence. Chin. J. Nat. Med. 2013, 11, 546-552. [CrossRef]

9. Pan, Y.; Zhang, X.; Wang, H.; Liang, Y.; Zhu, J.; Li, H.; Zhang, Z.; Wu, Q. Antioxidant potential of ethanolic extract of Polygonum cuspidatum and application in peanut oil. Food Chem. 2007, 105, 1518-1524. [CrossRef]

10. Wang, T.H.; Zhang, J.; Qiu, X.H.; Bai, J.Q.; Gao, Y.H.; Xu, W. Application of ultra-high-performance liquid chromatography coupled with LTQ-orbitrap mass spectrometry for the qualitative and quantitative analysis of Polygonum multiflorum Thumb. and its processed products. Molecules 2016, 21, 40. [CrossRef]

11. Fu, J.; Wang, M.; Guo, H.; Tian, Y.; Zhang, Z.; Song, R. Profiling of components of rhizoma et radix polygoni cuspidati by high-performance liquid chromatography with ultraviolet diode-array detector and ion trap/time-of-flight mass spectrometric detection. Pharmacogn. Mag. 2015, 11, 486-501.

12. Rue, E.A.; Rush, M.D.; van Breemen, R.B. Procyanidins: A comprehensive review encompassing structure elucidation via mass spectrometry. Phytochem. Rev. 2018, 17, 1-16. [CrossRef]

13. Rockenbach, I.I.; Jungfer, E.; Ritter, C.; Santiago-Schübel, B.; Thiele, B.; Fett, R.; Galensa, R. Characterization of flavan-3-ols in seeds of grape pomace by CE, HPLC-DAD-MS n and LC-ESI-FTICR-MS. Food Res. Int. 2012, 48, 848-855. [CrossRef]

14. Hellström, J.; Sinkkonen, J.; Karonen, M.; Mattila, P. Isolation and structure elucidation of procyanidin oligomers from Saskatoon berries (Amelanchier alnifolia). J. Agric. Food Chem. 2007, 55, 157-164. [CrossRef]

15. Glavnik, V.; Vovk, I.; Albreht, A. High performance thin-layer chromatography-mass spectrometry of Japanese knotweed flavan-3-ols and proanthocyanidins on silica gel plates. J. Chromatogr. A 2017, 1482, 97-108. [CrossRef]

16. Fan, P.; Terrier, L.; Hay, A.; Marston, A.; Hostettmann, K. Antioxidant and enzyme inhibition activities and chemical profiles of Polygonum sachalinensis F. Schmidt ex Maxim (Polygonaceae). Fitoterapia 2010, 81, 124-131. [CrossRef]

17. Hwang, J.T.; Kim, Y.; Jang, H.J.; Oh, H.M.; Lim, C.H.; Lee, S.W.; Rho, M.C. Study of the UV light conversion of feruloyl amides from Portulaca oleracea and their inhibitory effect on IL-6-induced STAT3 activation. Molecules 2016, 21, 865. [CrossRef]

18. Said, R.B.; Hamed, A.I.; Mahalel, U.A.; Al-Ayed, A.S.; Kowalczyk, M.; Moldoch, J.; Oleszek, W.; Stochmal, A. Tentative characterization of polyphenolic compounds in the male flowers of Phoenix dactylifera by liquid chromatography coupled with mass spectrometry and DFT. Int. J. Mol. Sci. 2017, 18, 512.

19. Zheng, C.; Hu, C.; Ma, X.; Peng, C.; Zhang, H.; Qin, L. Cytotoxic phenylpropanoid glycosides from Fagopyrum tataricum (L.) Gaertn. Food Chem. 2012, 132, 433-438. [CrossRef]

20. Wang, L.; Sang, M.; Liu, E.; Banahene, P.O.; Zhang, Y.; Wang, T.; Han, L.; Gao, X. Rapid profiling and pharmacokinetic studies of major compounds in crude extract from Polygonum multiflorum by UHPLC-Q-TOF-MS and UPLC-MS/MS. J. Pharm. Biomed. Anal. 2017, 140, 45-61. [CrossRef] 
21. Xu, W.; Zhang, J.; Huang, Z.; Qiu, X. Identification of new dianthrone glycosides from Polygonum multiflorum Thunb. using high-performance liquid chromatography coupled with LTQ-Orbitrap mass spectrometry detection: A strategy for the rapid detection of new low abundant metabolites from tradi. Anal. Methods 2012, 4, 1806-1812. [CrossRef]

22. Evtuguin, D.V.; Amado, F.M.L. Application of electrospray ionization mass spectrometry to the elucidation of the primary structure of lignin. Macromol. Biosci. 2003, 3, 339-343. [CrossRef]

23. Fan, P.; Hay, A.E.; Marston, A.; Lou, H.; Hostettmann, K. Chemical variability of the invasive neophytes Polygonum cuspidatum Sieb. and Zucc. and Polygonum sachalinensis F. Schmidt ex Maxim. Biochem. Syst. Ecol. 2009, 37, 24-34. [CrossRef]

24. Morreel, K. Profiling of oligolignols reveals monolignol coupling conditions in lignifying poplar xylem. Plant Physiol. 2004, 136, 3537-3549. [CrossRef]

25. Banoub, J.; Delmas, G.H.; Joly, N.; Mackenzie, G.; Cachet, N.; Benjelloun-Mlayah, B.; Delmas, M. A critique on the structural analysis of lignins and application of novel tandem mass spectrometric strategies to determine lignin sequencing. J. Mass Spectrom. 2015, 50, 5-48. [CrossRef]

26. Takasaki, M.; Kuroki, S.; Kozuka, M.; Konoshima, T. New phenylpropanoid esters of sucrose from Polygonum lapathifolium. J. Nat. Prod. 2001, 64, 1305-1308. [CrossRef]

27. Chen, X.; Wang, R.; Liu, B. An update on oligosaccharides and their esters from traditional chinese medicines: Chemical structures and biological activities. Evid. Based. Complement. Alternat. Med. 2015, 2015, 512675. [CrossRef]

28. Van Kiem, P.; Nhiem, N.X.; Cuong, N.X.; Hoa, T.Q.; Huong, H.T.; Huong, L.M.; Van Minh, C.; Kim, Y.H. New phenylpropanoid esters of sucrose from Polygonum hydropiper and their antioxidant activity. Arch. Pharm. Res. 2008, 31, 1477-1482. [CrossRef]

29. Ozarowski, M.; Piasecka, A.; Paszel-Jaworska, A.; Chaves, D.S.D.A.; Romaniuk, A.; Rybczynska, M.; Gryszczynska, A.; Sawikowska, A.; Kachlicki, P.; Mikolajczak, P.L.; et al. Comparison of bioactive compounds content in leaf extracts of Passiflora incarnata, P. caerulea and P. alata and in vitro cytotoxic potential on leukemia cell lines. Braz. J. Pharmacogn. 2018, 28, 179-191. [CrossRef]

30. Nguyen, T.K.O.; Jamali, A.; Grand, E.; Morreel, K.; Marcelo, P.; Gontier, E.; Dauwe, R. Phenylpropanoid profiling reveals a class of hydroxycinnamoyl glucaric acid conjugates in Isatis tinctoria leaves. Phytochemistry 2017, 144, 127-140. [CrossRef]

31. Zhao, Y.; Lee, M.-J.; Cheung, C.; Ju, J.-H.; Chen, Y.-K.; Liu, B.; Hu, L.-Q.; Yang, C.S. Analysis of multiple metabolites of tocopherols and tocotrienols in mice and humans. J. Agric. Food Chem. 2010, 58, 4844-4852. [CrossRef]

32. Mechelke, M.; Herlet, J.; Benz, J.P.; Schwarz, W.H.; Zverlov, V.V.; Liebl, W.; Kornberger, P. HPAEC-PAD for oligosaccharide analysis-Novel insights into analyte sensitivity and response stability. Anal. Bioanal. Chem. 2017, 409, 7169-7181. [CrossRef] [PubMed]

33. Ye, M.; Han, J.; Chen, H.; Zheng, J.; Guo, D. Analysis of phenolic compounds in rhubarbs using liquid chromatography coupled with electrospray ionization mass spectrometry. J. Am. Soc. Mass Spectrom. 2007, 18, 82-91. [CrossRef]

34. Wang, G.Y.; Chen, F.F.; Shi, Y.P. Ultra-performance LC-photodiode array-e $\lambda$-ESI-MS/MS screening method for the detection of radical-scavenging natural antioxidants from Radix et Rhizoma Rhei. J. Sep. Sci. 2011, 34, 268-277. [CrossRef] [PubMed]

35. Doherty, W.O.S.; Mousavioun, P.; Fellows, C.M. Value-adding to cellulosic ethanol: Lignin polymers. Ind. Crops Prod. 2011, 33, 259-276. [CrossRef]

36. Matsuda, S.; Kadota, S.; Tai, T.; Kikuchi, T. Isolation and structures of hedyotisol-A, -B, and -C novel dilignans from Hedyotis lawsoniae. Chem. Pharm. Bull. 1984, 32, 5066-5069. [CrossRef]

37. Jaiswal, R.; Sovdat, T.; Vivan, F.; Kuhnert, N. Profiling and characterization by LC-MSn of the chlorogenic acids and hydroxycinnamoylshikimate esters in Mateé (Ilex paraguariensis). J. Agric. Food Chem. 2010, 58, 5471-5484. [CrossRef] [PubMed]

38. Karaköse, H.; Jaiswal, R.; Kuhnert, N. Characterization and quantification of hydroxycinnamate derivatives in Stevia rebaudiana leaves by LC-MSn. J. Agric. Food Chem. 2011, 59, 10143-10150. [CrossRef] [PubMed] 
39. Jaiswal, R.; Matei, M.F.; Golon, A.; Witt, M.; Kuhnert, N. Understanding the fate of chlorogenic acids in coffee roasting using mass spectrometry based targeted and non-targeted analytical strategies. Food Funct. 2012, 3, 976-984. [CrossRef]

40. Dalzell, S.A.; Kerven, G.L. A rapid method for the measurement of Leucaena spp. proanthocyanidins by the proanthocyanidin (butanol/HCl) assay. J. Sci. Food Agric. 1998, 78, 405-416. [CrossRef]

41. Scalbert, A. Quantitative methods for the estimation of tannins in plant tissues. In Plant Polyphenols; Hemingway, R.W., Laks, P.E., Eds.; Plenum Press: New York, NY, USA, 1992; Volume 59, pp. 259-280.

42. Mikulski, D.; Molski, M. Quantitative structure-antioxidant activity relationship of trans-resveratrol oligomers, trans-4,4'-dihydroxystilbene dimer, trans-resveratrol-3-O-glucuronide, glucosides: Trans-piceid, cis-piceid, trans-astringin and trans-resveratrol-4'-O- $\beta$-D-glucopyranoside. Eur. J. Med. Chem. 2010, 45, 2366-2380. [PubMed]

43. Wenzel, E.; Soldo, T.; Erbersdobler, H.; Somoza, V. Bioactivity and metabolism of trans-resveratrol orally administered to Wistar rats. Mol. Nutr. Food Res. 2005, 49, 482-494. [CrossRef] [PubMed]

44. Lachowicz, S.; Oszmiański, J.; Wojdyło, A.; Cebulak, T.; Hirnle, L.; Siewiński, M. UPLC-PDA-Q/TOF-MS identification of bioactive compounds and on-line UPLC-ABTS assay in Fallopia japonica Houtt. and Fallopia sachalinensis Schmidt leaves and rhizomes grown in Poland. Eur. Food Res. Technol. 2019, 245, 691-706. [CrossRef]

45. Cos, P.; Bruyne, T.D.; Hermans, N.; Apers, S.; Berghe, D.V.; Vlietinck, A.J. Proanthocyanidins in health care: Current and new trends. Curr. Med. Chem. 2004, 11, 1345-1359. [CrossRef]

46. Koleckar, V.; Kubikova, K.; Rehakova, Z.; Kuca, K.; Jun, D.; Jahodar, L.; Opletal, L. Condensed and hydrolysable tannins as antioxidants influencing the health. Mini Rev. Med. Chem. 2008, 8, 436-447. [CrossRef] [PubMed]

47. Castellain, R.C.L.; Gesser, M.; Tonini, F.; Schulte, R.V.; Demessiano, K.Z.; Wolff, F.R.; Delle-Monache, F.; Netz, D.J.A.; Cechinel-Filho, V.; de Freitas, R.A.; et al. Chemical composition, antioxidant and antinociceptive properties of Litchi chinensis leaves. J. Pharm. Pharmacol. 2014, 66, 1796-1807. [CrossRef]

48. Soobrattee, M.A.; Neergheen, V.S.; Luximon-Ramma, A.; Aruoma, O.I.; Bahorun, T. Phenolics as potential antioxidant therapeutic agents: Mechanism and actions. Mutat. Res. 2005, 579, 200-213. [CrossRef]

49. Prieto, P.; Pineda, M.; Aguilar, M. Spectrophotometric quantitation of antioxidant capacity through the formation of a phosphomolybdenum complex: Specific application to the determination of vitamin E. Anal. Biochem. 1999, 269, 337-341. [CrossRef]

50. Luo, L.; Cui, Y.; Cheng, J.; Fang, B.; Wei, Z.; Sun, B. An approach for degradation of grape seed and skin proanthocyanidin polymers into oligomers by sulphurous acid. Food Chem. 2018, 256, 203-211. [CrossRef]

51. Dallas, C.; Hipolito-Reis, P.; Ricardo-da-Silva, J.M.; Laureano, O. Influence of acetaldehyde, pH, and temperature on transformation of procyanidins in model wine solutions. Am. J. Enol. Vitic. 2003, 54, 119-124.

52. Fan, P.; Marston, A.; Hay, A.E.; Hostettmann, K. Rapid separation of three glucosylated resveratrol analogues from the invasive plant Polygonum cuspidatum by high-speed countercurrent chromatography. J. Sep. Sci. 2009, 32, 2979-2984. [CrossRef]

53. Zhang, D.; Li, X.; Hao, D.; Li, G.; Xu, B.; Ma, G.; Su, Z. Systematic purification of polydatin, resveratrol and anthraglycoside B from Polygonum cuspidatum Sieb. et Zucc. Sep. Purif. Technol. 2009, 66, 329-339. [CrossRef]

54. Fan, P.; Hostettmann, K.; Lou, H. Allelochemicals of the invasive neophyte Polygonum cuspidatum Sieb. \& Zucc. (Polygonaceae). Chemoecology 2010, 20, 223-227.

55. Yang, F.; Zhang, T.; Ito, Y. Large-scale separation of resveratrol, anthraglycoside A and anthraglycoside B from Polygonum cuspidatum Sieb. et Zucc by high-speed counter-current chromatography. J. Chromatogr. A 2001, 919, 443-448. [CrossRef]

56. Chu, X.; Sun, A.; Liu, R. Preparative isolation and purification of five compounds from the Chinese medicinal herb Polygonum cuspidatum Sieb. et Zucc. by high-speed counter-current chromatography. J. Chromatogr. A 2005, 1097, 33-39. [CrossRef]

57. Pharmacopoeia of China. Part 1; China Medical Science and Technology Press: Beijing, China, 2005; pp. $180-181$.

58. Brand-Williams, W.; Cuvelier, M.E.; Berset, C. Use of a free radical method to evaluate antioxidant activity. LWT Food Sci. Technol. 1995, 28, 25-30. [CrossRef]

59. Woźniak, D.; Dryś, A.; Matkowski, A. Antiradical and antioxidant activity of flavones from Scutellariae baicalensis radix. Nat. Prod. Res. 2015, 29, 1567-1570. [CrossRef] 
60. Nawrot-Hadzik, I.; Granica, S.; Abel, R.; Czapor-Irzabek, H.; Matkowski, A. Analysis of antioxidant polyphenols in loquat leaves using HPLC-based activity profiling. Nat. Prod. Commun. 2016, 12, 163-166.

61. Li, Y.G.; Tanner, G.; Larkin, P. The DMACA Protocol and the threshold proanthocyanidin content for bloat safety in forage legumes. J. Sci. Food Agric. 1996, 70, 89. [CrossRef]

Sample Availability: Samples of the compounds are available from the first author (I.N.-H.) upon request.

(C) 2019 by the authors. Licensee MDPI, Basel, Switzerland. This article is an open access article distributed under the terms and conditions of the Creative Commons Attribution (CC BY) license (http:/ / creativecommons.org/licenses/by/4.0/). 